\title{
R-Regularity of Set-Valued Mappings Under the Relaxed Constant Positive Linear Dependence Constraint Qualification with Applications to Parametric and Bilevel Optimization
}

\author{
Patrick Mehlitz ${ }^{1}$ (D) . Leonid I. Minchenko² (D)
}

Received: 14 May 2020 / Accepted: 21 February 2021 /Published online: 15 June 2021

(C) The Author(s) 2021

\begin{abstract}
The presence of Lipschitzian properties for solution mappings associated with nonlinear parametric optimization problems is desirable in the context of, e.g., stability analysis or bilevel optimization. An example of such a Lipschitzian property for set-valued mappings, whose graph is the solution set of a system of nonlinear inequalities and equations, is Rregularity. Based on the so-called relaxed constant positive linear dependence constraint qualification, we provide a criterion ensuring the presence of the R-regularity property. In this regard, our analysis generalizes earlier results of that type which exploited the stronger Mangasarian-Fromovitz or constant rank constraint qualification. Afterwards, we apply our findings in order to derive new sufficient conditions which guarantee the presence of Rregularity for solution mappings in parametric optimization. Finally, our results are used to derive an existence criterion for solutions in pessimistic bilevel optimization and a sufficient condition for the presence of the so-called partial calmness property in optimistic bilevel optimization.
\end{abstract}

Keywords Bilevel optimization · Parametric optimization - Partial calmness - RCPLD · R-regularity

Mathematics Subject Classification 2010 49J53 · 90C30 · 90C31

Patrick Mehlitz

mehlitz@b-tu.de

Leonid I. Minchenko

leonidm@insoftgroup.com

1 Brandenburgische Technische Universität Cottbus-Senftenberg, Institute of Mathematics, 03046 Cottbus, Germany

2 Belarus State University of Informatics and Radioelectronics, 6 P. Brovki Street, Minsk 220013, Belarus 


\section{Introduction}

Lipschitzian properties of implicitly given set-valued mappings are of essential importance in order to study the stability of optimization problems, see e.g. [25, 32, 37] and the references therein. Particularly, such stability is desirable in the context of bilevel optimization where a function has to be minimized over the graph of a solution mapping associated with a given parametric optimization problem, see $[5,12,16]$ or Section 4.2 for details. Indeed, in order to infer existence results, optimality conditions, or solution algorithms in bilevel programming, one generally has to assume the presence of certain properties of this solution map. However, it is often not easy to verify such properties. In this paper, we focus on the derivation of sufficient criteria for the presence of so-called $R$-regularity of setvalued mappings, see Definition 2.2. This property, in turn, is beneficial in order to study Lipschitzian properties of marginal (or optimal value) functions and solution mappings in parametric optimization, see $[6,32,36]$, and these features possess some extensions to bilevel optimization as well.

In this paper, we investigate set-valued mappings $\Gamma: \mathbb{R}^{n} \rightrightarrows \mathbb{R}^{m}$ of the form

$$
\forall x \in \mathbb{R}^{n}: \quad \Gamma(x):=\left\{\begin{array}{l|l}
y \in \mathbb{R}^{m} & \begin{array}{l}
h_{i}(x, y) \leq 0 \\
h_{i}(x, y)=0
\end{array} \quad i \in J
\end{array}\right\}
$$

where $I:=\{1, \ldots, \ell\}$ and $J:=\{\ell+1, \ldots, p\}$ are index sets and $h_{1}, \ldots, h_{p}: \mathbb{R}^{n} \times \mathbb{R}^{m} \rightarrow \mathbb{R}$ are given functions. Precise assumptions on the continuity and smoothness properties of $h_{1}, \ldots, h_{p}$ will be specified in the course of the paper. It is well known that the presence of R-regularity for mappings of this type is guaranteed under validity of the MangasarianFromovitz constraint qualification, see [8,32]. More recently, this result has been extended to situations where relaxed versions of the constant rank constraint qualification hold at the underlying reference points, see $[6,36]$. However, in some situations, these qualification conditions may turn out to be too selective when investigating the presence of R-regularity for solution mappings, see e.g. Remark 4.2. That is why we aim for a generalization of these findings in the presence of the so-called relaxed constant positive linear dependence constraint qualification, introduced in [1], which is generally weaker than the aforementioned qualification conditions. Our main results Theorems 3.2 and 3.3 depict that this is indeed possible. With these new sufficient conditions for the presence of R-regularity for the mapping $\Gamma$ at hand, we are in position to state new criteria ensuring local Lipschitz continuity of the marginal function and R-regularity of the solution mapping associated with nonlinear parametric optimization problems whose feasible region is modeled with the aid of $\Gamma$. Afterwards, we use these findings in order to study the existence of so-called pessimistic solutions as well as the presence of the celebrated partial calmness property in bilevel optimization. The latter, introduced in [46], is one of the key assumptions one generally postulates on the optimal value reformulation of an optimistic bilevel optimization problem in order to infer necessary optimality conditions and solution algorithms, see Section 4.2 for details and suitable references.

The remaining parts of this manuscript are organized as follows: In Section 2, we provide the fundamental notation exploited in this paper. Furthermore, we recall some important constraint qualifications from nonlinear programming as well as the underlying fundamentals of set-valued analysis. Section 3 is dedicated to the study of the relaxed constant positive linear dependence constraint qualification as a sufficient condition for R-regularity of the mapping $\Gamma$. In Section 4, we investigate some applications of our findings. First, we apply the obtained results to nonlinear parametric optimization problems in order to state new 
sufficient conditions for the local Lipschitz continuity of the associated optimal value function as well as R-regularity of the associated solution mapping in Section 4.1. Afterwards, we employ these results in the context of bilevel optimization in order to formulate criteria ensuring the existence of pessimistic solutions as well as the presence of partial calmness in Section 4.2. In Section 5, we close the paper with the aid of some final comments.

\section{Notation and Preliminaries}

In this paper, we mainly make use of standard notation. The tools of set-valued analysis we exploit here can be found, e.g., in [4, 37, 42].

\subsection{Basic Notation}

Throughout the paper, we equip $\mathbb{R}^{n}$ with the Euclidean norm $\|\cdot\|$. For some point $x \in \mathbb{R}^{n}$ and a scalar $\varepsilon>0$, we use

$$
\mathbb{U}_{\varepsilon}(x):=\left\{y \in \mathbb{R}^{n} \mid\|y-x\|<\varepsilon\right\}, \quad \mathbb{B}_{\varepsilon}(x):=\left\{y \in \mathbb{R}^{n} \mid\|y-x\| \leq \varepsilon\right\}
$$

in order to denote the open and closed $\varepsilon$-ball around $x$, respectively. For brevity, we make use of $\mathbb{B}:=\mathbb{B}_{1}(0)$. For a nonempty and closed set $A \subset \mathbb{R}^{n}$, we use

$$
\operatorname{dist}(x, A):=\inf \{\|y-x\| \mid y \in A\}, \quad \Pi(x, A):=\operatorname{argmin}\{\|y-x\| \mid y \in A\}
$$

to denote the distance of $x$ to $A$ and the set of projections of $x$ onto $A$, respectively. It is well known that the distance function $\operatorname{dist}(\cdot, A): \mathbb{R}^{n} \rightarrow \mathbb{R}$ is Lipschitz continuous with Lipschitz modulus 1 . Generally, we call a map $\phi: \mathbb{R}^{n} \rightarrow \mathbb{R}^{m}$ locally Lipschitz continuous at $x$ w.r.t. $\Omega \subset \mathbb{R}^{n}$ whenever there are $\delta>0$ and $L>0$ such that

$$
\forall y, y^{\prime} \in \mathbb{U}_{\delta}(x) \cap \Omega: \quad\left\|\phi(y)-\phi\left(y^{\prime}\right)\right\| \leq L\left\|y-y^{\prime}\right\|
$$

holds. Note that this notion is only reasonable in the situation $x \in \operatorname{cl} \Omega$. For $\Omega:=\mathbb{R}^{n}$, we recover the classical definition of local Lipschitz continuity.

Let $I_{1}$ as well as $I_{2}$ be finite index sets and let $\left(a^{i}\right)_{i \in I_{1}} \subset \mathbb{R}^{n}$ as well as $\left(b^{i}\right)_{i \in I_{2}} \subset \mathbb{R}^{n}$ be two given families of vectors. We call the pair of families $\left(\left(a^{i}\right)_{i \in I_{1}},\left(b^{i}\right)_{i \in I_{2}}\right)$ positivelinearly dependent whenever there are scalars $\alpha_{i} \geq 0, i \in I_{1}$, and $\beta_{i}, i \in I_{2}$, which are not all vanishing such that

$$
\sum_{i \in I_{1}} \alpha_{i} a^{i}+\sum_{i \in I_{2}} \beta_{i} b^{i}=0
$$

Otherwise, we refer to this pair of families as positive-linearly independent. A family of vectors $\left(a^{i}\right)_{i \in I_{1}}$ is called positive-linearly dependent (independent) whenever the pair of families $\left(\left(a^{i}\right)_{i \in I_{1}}, \varnothing\right)$ is positive-linearly dependent (independent).

The following lemma follows from [1, Lemma 1].

Lemma 2.1 Let $v^{1}, \ldots, v^{r+s} \in \mathbb{R}^{n}$ be given vectors such that the family $\left(v^{i}\right)_{i=1}^{r}$ is linearly independent. Furthermore, let $z \in \mathbb{R}^{n} \backslash\{0\}$ be given as $z=\sum_{i=1}^{r+s} \alpha_{i} v^{i}$ for reals $\alpha_{1}, \ldots, \alpha_{r+s}$ satisfying $\alpha_{r+1}, \ldots, \alpha_{r+s}>0$. Then there exist an index set $\mathcal{I} \subset$ $\{r+1, \ldots, r+s\}$ and reals $\bar{\alpha}_{i}, i \in\{1, \ldots, r\} \cup \mathcal{I}$, satisfying $\bar{\alpha}_{i}>0$ for all $i \in \mathcal{I}$, such that

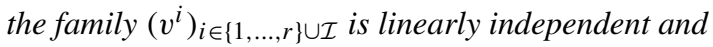

$$
z=\sum_{i \in\{1, \ldots, r\} \cup \mathcal{I}} \bar{\alpha}_{i} v^{i} .
$$




\subsection{Constraint Qualifications in Nonlinear Programming}

Supposing that $\Gamma$ models the feasible region of a given parametric optimization problem, certain constraint qualifications need to be imposed on the images of $\Gamma$ in order to ensure that the associated Karush-Kuhn-Tucker conditions provide a necessary optimality condition. In this regard, we postulate the following assumption which may hold throughout the section.

Assumption 2.1 Let us fix a reference parameter $\bar{x} \in \mathbb{R}^{n}$ and some point $\bar{y} \in \Gamma(\bar{x})$. Furthermore, let all the functions $h_{1}, \ldots, h_{p}$ be continuous as well as continuously differentiable w.r.t. $y$ in a neighborhood of $\{\bar{x}\} \times \Gamma(\bar{x})$.

Let us now introduce the qualification conditions of our interest. Therefore, we will exploit the set of indices associated with inequality constraints active at $(\bar{x}, \bar{y})$ which is defined as stated below:

$$
I(\bar{x}, \bar{y}):=\left\{i \in I \mid h_{i}(\bar{x}, \bar{y})=0\right\} .
$$

Definition 2.1 We say that

(a) the linear independence constraint qualification (LICQ) holds at $(\bar{x}, \bar{y})$ whenever the family $\left(\nabla_{y} h_{i}(\bar{x}, \bar{y})\right)_{i \in I(\bar{x}, \bar{y}) \cup J}$ is linearly independent,

(b) the Mangasarian-Fromovitz constraint qualification (MFCQ) holds at $(\bar{x}, \bar{y})$ whenever the pair of families

$$
\left(\left(\nabla_{y} h_{i}(\bar{x}, \bar{y})\right)_{i \in I(\bar{x}, \bar{y})},\left(\nabla_{y} h_{i}(\bar{x}, \bar{y})\right)_{i \in J}\right)
$$

is positive-linearly independent,

(c) the relaxed constant rank constraint qualification (RCRCQ) holds at $(\bar{x}, \bar{y})$ (w.r.t. $\Omega \subset$ $\mathbb{R}^{n}$ ) whenever there is a neighborhood $U$ of $(\bar{x}, \bar{y})$ such that for each set $K \subset I(\bar{x}, \bar{y})$, the family $\left(\nabla_{y} h_{i}(x, y)\right)_{i \in K \cup J}$ has constant rank on $U$ (on $U \cap\left(\Omega \times \mathbb{R}^{m}\right)$ ),

(d) the relaxed constant positive linear dependence constraint qualification (RCPLD) holds at $(\bar{x}, \bar{y})$ (w.r.t. $\left.\Omega \subset \mathbb{R}^{n}\right)$ whenever there are a neighborhood $U$ of $(\bar{x}, \bar{y})$ and an index set $S \subset J$ such that the following conditions hold:

(i) $\left\{\nabla_{y} h_{i}(\bar{x}, \bar{y}) \mid i \in S\right\}$ is a basis of the span of $\left\{\nabla_{y} h_{i}(\bar{x}, \bar{y}) \mid i \in J\right\}$,

(ii) the family $\left(\nabla_{y} h_{i}(x, y)\right)_{i \in J}$ has constant rank on $U$ (on $U \cap\left(\Omega \times \mathbb{R}^{m}\right)$ ), and

(iii) for each set $K \subset I(\bar{x}, \bar{y})$ such that the pair of families

$$
\left(\left(\nabla_{y} h_{i}(\bar{x}, \bar{y})\right)_{i \in K},\left(\nabla_{y} h_{i}(\bar{x}, \bar{y})\right)_{i \in S}\right)
$$

is positive-linearly dependent, the family $\left(\nabla_{y} h_{i}(x, y)\right)_{i \in K \cup S}$ is linearly dependent for each point $(x, y) \in U$ (for each point $(x, y) \in U \cap\left(\Omega \times \mathbb{R}^{m}\right)$ ).

While LICQ and MFCQ are well-known constraint qualifications, RCRCQ and RCPLD are less popular. Let us mention that RCRCQ, which has been introduced in [35], is a less restrictive constraint qualification than the classical constant rank constraint qualification, see [30]. On the other hand, RCPLD dates back to [1] and generalizes the classical constant positive linear dependence constraint qualification, see [2, 40]. Checking these references, one can observe that both MFCQ and RCRCQ individually imply validity of RCPLD. However, neither does MFCQ imply validity of RCRCQ nor vice versa. Clearly, LICQ is stronger than MFCQ and RCRCQ. Let us mention that RCPLD is stable in the sense that whenever 
it is valid at some reference point, then it also holds in a neighborhood of this point. In order to see this, one may adapt the proof of [1, Theorem 4], which is stated in the non-parametric setting, to the situation at hand. Finally, we would like to mention that the notion of RCPLD can be extended to non-smooth constraint systems as well as complementarity-type feasible regions, and, thus, applies to mathematical programs with complementarity constraints and different reformulations of bilevel optimization problems, see [10, 26, 27, 44] for details.

\subsection{Properties of Set-Valued Mappings}

Let $\Upsilon: \mathbb{R}^{n} \rightrightarrows \mathbb{R}^{m}$ be a set-valued mapping. We refer to the sets

$$
\operatorname{gph} \Upsilon:=\left\{(x, y) \in \mathbb{R}^{n} \times \mathbb{R}^{m} \mid y \in \Upsilon(x)\right\}, \quad \operatorname{dom} \Upsilon:=\left\{x \in \mathbb{R}^{n} \mid \Upsilon(x) \neq \varnothing\right\}
$$

as graph and domain of $\Upsilon$, respectively. Let us fix a point $\bar{x} \in \operatorname{dom} \Upsilon$. We call $\Upsilon$ locally bounded at $\bar{x}$ whenever there are a bounded set $B \subset \mathbb{R}^{m}$ and a neighborhood $U \subset \mathbb{R}^{n}$ of $\bar{x}$ such that $\Upsilon(x) \subset B$ holds for all $x \in U$. One calls $\Upsilon$ upper semicontinuous at $\bar{x}$ whenever for each open set $O \subset \mathbb{R}^{m}$ which satisfies $\Upsilon(\bar{x}) \subset O$, there exists a neighborhood $U \subset \mathbb{R}^{n}$ of $\bar{x}$ such that $\Upsilon(x) \subset O$ holds for all $x \in U$. Recall that $\Upsilon$ is called lower semicontinuous at $\bar{x}$ (w.r.t. $\Omega \subset \mathbb{R}^{n}$ ) whenever for each open set $O \subset \mathbb{R}^{m}$ with $\Upsilon(\bar{x}) \cap O \neq \varnothing$, there is a neighborhood $U \subset \mathbb{R}^{n}$ of $\bar{x}$ such that $\Upsilon(x) \cap O \neq \varnothing$ holds for all $x \in U$ (for all $x \in U \cap \Omega$ ). We call $\Upsilon$ inner semicontinuous at some point $(\bar{x}, \bar{y}) \in \operatorname{gph} \Upsilon$ (w.r.t. $\Omega$ ) whenever for each sequence $\left\{x^{k}\right\}_{k \in \mathbb{N}} \subset \mathbb{R}^{n}\left(\left\{x^{k}\right\}_{k \in \mathbb{N}} \subset \Omega\right)$ converging to $\bar{x}$, there exists a sequence $\left\{y^{k}\right\}_{k \in \mathbb{N}} \subset \mathbb{R}^{m}$ which converges to $\bar{y}$ and satisfies $y^{k} \in \Upsilon\left(x^{k}\right)$ for sufficiently large $k \in \mathbb{N}$. Note that $\Upsilon$ is lower semicontinuous at $\bar{x}$ (w.r.t. $\Omega$ ) if and only if it is inner semicontinuous at each point from $\{\bar{x}\} \times \Upsilon(\bar{x})$ (w.r.t. $\Omega$ ). The situation $\Omega:=\operatorname{dom} \Upsilon$ will be of particular interest in this manuscript.

In the theory of set-valued analysis, there exist several different notions of Lipschitzianity. Recall that $\Upsilon$ possesses the Aubin property at some point $(\bar{x}, \bar{y}) \in \operatorname{gph} \Upsilon$ (w.r.t. $\Omega$ ) whenever there exist neighborhoods $U$ and $V$ of $\bar{x}$ and $\bar{y}$, respectively, as well as a constant $\kappa>0$ such that

$$
\forall x, x^{\prime} \in U\left(\forall x, x^{\prime} \in U \cap \Omega\right): \quad \Upsilon(x) \cap V \subset \Upsilon\left(x^{\prime}\right)+\kappa\left\|x-x^{\prime}\right\| \mathbb{B}
$$

holds. One can easily check that whenever $\Upsilon$ possesses the Aubin property at $(\bar{x}, \bar{y})$ (w.r.t. $\Omega$ ), then it is inner semicontinuous (w.r.t. $\Omega$ ) at this point. Using the concept of coderivatives which is based on the limiting normal cone from variational analysis, one can formulate a necessary and sufficient condition for the presence of the Aubin property for set-valued mappings with closed graphs, see [37, Theorem 4.10]. In [37, Corollary 4.39], one can find a characterization of the Aubin property of $\Gamma$ from (1.1) at some point of its graph under validity of an MFCQ-type assumption. Let us, however, note that MFCQ from Definition 2.1 is only sufficient but not necessary for the presence of the Aubin property. A recent study on the presence of the Aubin property for implicitly defined set-valued mappings of more general form can be found in [25].

Let us now focus on the particular mapping $\Gamma$ from (1.1) in more detail. In this manuscript, we are interested in the property of $\Gamma$ being so-called R-regular at a point of its graph, see [32, Section 6.2]. 
Definition 2.2 Fix $(\bar{x}, \bar{y}) \in \operatorname{gph} \Gamma$ and some set $\Omega \subset \mathbb{R}^{n}$. Then $\Gamma$ is called $R$-regular at $(\bar{x}, \bar{y})$ (w.r.t. $\Omega$ ) whenever there exist a constant $\kappa>0$ and a neighborhood $U$ of $(\bar{x}, \bar{y})$ such that the condition

$$
\begin{aligned}
& \forall(x, y) \in U\left(\forall(x, y) \in U \cap\left(\Omega \times \mathbb{R}^{m}\right)\right): \\
& \quad \operatorname{dist}(y, \Gamma(x)) \leq \kappa \max \left\{0, \max \left\{h_{i}(x, y) \mid i \in I\right\}, \max \left\{\left|h_{i}(x, y)\right| \mid i \in J\right\}\right\}
\end{aligned}
$$

holds.

The notion of R-regularity can be traced back to [21,28] where it has been exploited as a constraint qualification. Following [9, 20,41], one might be tempted to say that the presence of R-regularity is equivalent to the validity of a local error bound condition at some reference point of the constraint system induced by $\Gamma$ provided the latter does not depend on the parameter. In this regard, R-regularity of a parametric constraint system is a generalization of the concept of error bounds. We refer the interested reader to [45] where the concept of socalled uniform parametric error bounds, which is closely related to R-regularity, is studied. Let us note that due to [41, Theorem 1] or [8, Theorem 3.2], R-regularity of $\Gamma$ at a given reference point is implied by validity of MFCQ at the latter. A generalization of this result to the setting where the functions $h_{1}, \ldots, h_{p}$ are non-smooth can be found in [48]. We would like to point out that R-regularity can be interpreted as a variant of metric regularity, see [29] and the references therein, and is stronger than metric subregularity of the feasibility mapping associated with the given parametric constraint set where the parameter is fixed, see [24, Section 1]. Furthermore, following [24, 41], it is possible to generalize the concept of R-regularity, which is called stability or Robinson stability in these papers, to geometric constraint systems of the type

$$
\tilde{h}(x, y) \in C
$$

where $\tilde{h}: \mathbb{R}^{n} \times \mathbb{R}^{m} \rightarrow \mathbb{R}^{p}$ is continuously differentiable w.r.t. $y$ and $C \subset \mathbb{R}^{p}$ is a closed set.

Invoking [6, Theorem 5.1], one can easily check that whenever $\Gamma$ is R-regular at $(\bar{x}, \bar{y})$ w.r.t. $\Omega$ while all the functions $h_{1}, \ldots, h_{p}$ are locally Lipschitz continuous at this point, then $\Gamma$ possesses the Aubin property w.r.t. $\Omega$ at this point. By means of simple examples, one can check that the converse statement does not hold in general even if the data functions are continuously differentiable and, thus, locally Lipschitzian, see [36, Example 1]. The following result even holds in the absence of local Lipschitz continuity of the data functions.

Lemma 2.2 Let $\Gamma$ be R-regular at some point $(\bar{x}, \bar{y}) \in \operatorname{gph} \Gamma$ w.r.t. dom $\Gamma$. Furthermore, let the functions $h_{1}, \ldots, h_{p}$ be continuous at $(\bar{x}, \bar{y})$ and let $h_{1}(x, \cdot), \ldots, h_{p}(x, \cdot): \mathbb{R}^{m} \rightarrow \mathbb{R}$ be continuous for each $x \in \operatorname{dom} \Gamma$ which comes from a neighborhood of $\bar{x}$. Then $\Gamma$ is inner semicontinuous at $(\bar{x}, \bar{y})$ w.r.t. $\operatorname{dom} \Gamma$.

Proof The assumptions of the lemma particularly imply the existence of a constant $\kappa>0$ and some $\delta>0$ such that

$$
\operatorname{dist}(\bar{y}, \Gamma(x)) \leq \kappa \max \left\{0, \max \left\{h_{i}(x, \bar{y}) \mid i \in I\right\}, \max \left\{\left|h_{i}(x, \bar{y})\right| \mid i \in J\right\}\right\}
$$

holds for all $x \in \mathbb{U}_{\delta}(\bar{x}) \cap \operatorname{dom} \Gamma$. Thus, for each sequence $\left\{x^{k}\right\}_{k \in \mathbb{N}} \subset \operatorname{dom} \Gamma$ with $x^{k} \rightarrow \bar{x}$, the estimate

$$
\left\|\bar{y}-y^{k}\right\| \leq \kappa \max \left\{0, \max \left\{h_{i}\left(x^{k}, \bar{y}\right) \mid i \in I\right\}, \max \left\{\left|h_{i}\left(x^{k}, \bar{y}\right)\right| \mid i \in J\right\}\right\}
$$

holds for sufficiently large $k \in \mathbb{N}$ where $y^{k} \in \Pi\left(\bar{y}, \Gamma\left(x^{k}\right)\right)$ is arbitrarily chosen. Note that $\Pi\left(\bar{y}, \Gamma\left(x^{k}\right)\right)$ is nonempty for each $k \in \mathbb{N}$ since $\Gamma\left(x^{k}\right)$ is nonempty and closed by continuity of $h_{1}\left(x^{k}, \cdot\right), \ldots, h_{p}\left(x^{k}, \cdot\right)$ and the choice $x^{k} \in \operatorname{dom} \Gamma$ for sufficiently large $k \in$ 
$\mathbb{N}$. Exploiting the continuity of $h_{1}, \ldots, h_{p}$ at $(\bar{x}, \bar{y})$, we find $\left\|\bar{y}-y^{k}\right\| \rightarrow 0$ as $k \rightarrow \infty$, i.e., $\Gamma$ is inner semicontinuous at $(\bar{x}, \bar{y})$ w.r.t. $\operatorname{dom} \Gamma$.

By definition, R-regularity of a set-valued mapping at a given reference point is stable in the sense that it extends to points in a sufficiently small neighborhood. However, we get the following even stronger stability property from [32, Lemma 6.19] which shows that the modulus of R-regularity is uniformly bounded in a neighborhood of a compact set of points where a given set-valued mapping is R-regular.

Lemma 2.3 Let $C \subset \mathrm{gph} \Gamma$ be compact and assume that $\Gamma$ is $R$-regular w.r.t. $\operatorname{dom} \Gamma$ at each point from $C$. Furthermore, let $O$ be a neighborhood of $C$ where $h_{1}, \ldots, h_{p}$ are continuous. Then there exist a constant $\kappa>0$ and an open set $U$ such that $C \subset U \subset O$ while (2.1) holds with $\Omega:=\operatorname{dom} \Gamma$, i.e., there is a uniform modulus $\kappa$ of $R$-regularity on $C$.

\section{A Sufficient Condition for R-Regularity}

If not stated otherwise, we assume that Assumption 2.1 holds throughout the section. For simplicity, let us postulate that the functions $h_{i}(x, \cdot): \mathbb{R}^{m} \rightarrow \mathbb{R}, i \in I \cup J$, are continuous for each $x \in \operatorname{dom} \Gamma$. Finally, we will, at some instances, exploit the following additional assumptions.

(A1) For each $x \in \mathbb{R}^{n}$, the functions $h_{i}(x, \cdot): \mathbb{R}^{m} \rightarrow \mathbb{R}, i \in I$, are convex while the functions $h_{i}(x, \cdot): \mathbb{R}^{m} \rightarrow \mathbb{R}, i \in J$, are affine.

(A2) The set-valued mapping $\Gamma$ is locally bounded at $\bar{x} \in \operatorname{dom} \Gamma$.

Subsequently, we will first derive a sequential characterization of R-regularity which holds under validity of the aforementioned conditions. Afterwards, we will relate this sequential characterization with the validity of the constraint qualification RCPLD.

\subsection{A Sequential Characterization of R-Regularity}

For some parameter $x \in \operatorname{dom} \Gamma$ and $v \notin \Gamma(x), \Pi(v, \Gamma(x))$ equals the solution set of

$$
\min _{y}\{\|y-v\| \mid y \in \Gamma(x)\}
$$

since $\Gamma(x)$ is a closed set by continuity of $h_{1}(x, \cdot), \ldots, h_{p}(x, \cdot)$. Due to $v \notin \Gamma(x)$, the objective function of the above problem is continuously differentiable in a neighborhood of all points from $\Pi(v, \Gamma(x))$. Thus, it is reasonable to investigate the associated Lagrange multiplier set

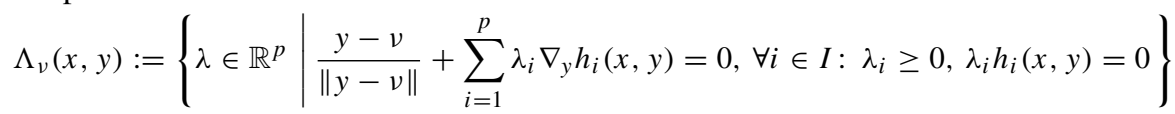

for each $y \in \Pi(v, \Gamma(x))$ as long as the pair $(x, y)$ is close to $\{\bar{x}\} \times \Gamma(\bar{x})$. For some constant $M>0$, we make use of

$$
\Lambda_{v}^{M}(x, y):=\left\{\lambda \in \Lambda_{v}(x, y)\left|\sum_{i=1}^{p}\right| \lambda_{i} \mid \leq M\right\} .
$$

Let us note that under validity of (A1), the image sets of $\Gamma$ are convex which yields that the associated projection sets from above are actually singletons.

Using this notation, we obtain the following technical lemma. 
Lemma 3.1 Let (A1) and (A2) hold. Assume that there exist a constant $M>0$ as well as sequences $\left\{x^{k}\right\}_{k \in \mathbb{N}} \subset \operatorname{dom} \Gamma,\left\{v^{k}\right\}_{k \in \mathbb{N}} \subset \mathbb{R}^{m}$, and $\left\{y^{k}\right\}_{k \in \mathbb{N}} \subset \mathbb{R}^{m}$ satisfying $x^{k} \rightarrow \bar{x}$, $v^{k} \rightarrow \bar{y}$, as well as $v^{k} \notin \Gamma\left(x^{k}\right)$ and $y^{k} \in \Pi\left(v^{k}, \Gamma\left(x^{k}\right)\right)$ for all $k \in \mathbb{N}$ such that the set $\Lambda_{v^{k}}^{M}\left(x^{k}, y^{k}\right)$ is nonempty for sufficiently large $k \in \mathbb{N}$. Then we have $y^{k} \rightarrow \bar{y}$ and

$$
\operatorname{dist}\left(v^{k}, \Gamma\left(x^{k}\right)\right) \leq M \max \left\{0, \max \left\{h_{i}\left(x^{k}, v^{k}\right) \mid i \in I\right\}, \max \left\{\left|h_{i}\left(x^{k}, v^{k}\right)\right| \mid i \in J\right\}\right\}
$$

for sufficiently large $k \in \mathbb{N}$.

Proof Due to (A2), $\left\{y^{k}\right\}_{k \in \mathbb{N}}$ is bounded. Fix an arbitrary convergent subsequence $\left\{y^{k_{s}}\right\}_{s \in \mathbb{N}}$ with limit $\tilde{y} \in \mathbb{R}^{m}$. By assumption, for all sufficiently large $s \in \mathbb{N}$, we find a multiplier $\lambda^{k_{s}} \in \Lambda_{\nu^{k_{s}}}^{M}\left(x^{k_{s}}, y^{k_{s}}\right)$. Exploiting (A1) and the definition of the set $\Lambda_{\nu^{k_{s}}}^{M}\left(x^{k_{s}}, y^{k_{s}}\right)$, we obtain

$$
\begin{aligned}
\left\|y^{k_{s}}-v^{k_{s}}\right\| & =\sum_{i=1}^{p} \lambda_{i}^{k_{s}} \nabla_{y} h_{i}\left(x^{k_{s}}, y^{k_{s}}\right)^{\top}\left(v^{k_{s}}-y^{k_{s}}\right) \\
& \leq \sum_{i=1}^{p} \lambda_{i}^{k_{s}}\left(\left(h_{i}\left(x^{k_{s}}, v^{k_{s}}\right)-h_{i}\left(x^{k_{s}}, y^{k_{s}}\right)\right)=\sum_{i=1}^{p} \lambda_{i}^{k_{s}} h_{i}\left(x^{k_{s}}, v^{k_{s}}\right)\right. \\
& \leq \sum_{i \in I} \lambda_{i}^{k_{s}} \max \left\{0, h_{i}\left(x^{k_{s}}, v^{k_{s}}\right)\right\}+\sum_{i \in J}\left|\lambda_{i}^{k_{s}}\right|\left|h_{i}\left(x^{k_{s}}, v^{k_{s}}\right)\right| \\
& \leq M \max \left\{0, \max \left\{h_{i}\left(x^{k_{s}}, v^{k_{s}}\right) \mid i \in I\right\}, \max \left\{\left|h_{i}\left(x^{k_{s}}, v^{k_{s}}\right)\right| \mid i \in J\right\}\right\}
\end{aligned}
$$

for sufficiently large $s \in \mathbb{N}$. Taking the limit $s \rightarrow \infty$ yields $\|\tilde{y}-\bar{y}\| \leq 0$, i.e., we find $\tilde{y}=\bar{y}$. Particularly, the bounded sequence $\left\{y^{k}\right\}_{k \in \mathbb{N}}$ possesses the unique accumulation point $\bar{y}$ which must be its limit. Reprising the above arguments, we infer the second statement of the lemma from $\operatorname{dist}\left(v^{k}, \Gamma\left(x^{k}\right)\right)=\left\|y^{k}-v^{k}\right\|$.

Next, we exploit Lemma 3.1 in order to characterize R-regularity of $\Gamma$ under validity of (A1) and (A2). This result is related to [6, Theorem 3.2] and [36, Theorems 2 and 3] where these assumptions are replaced by some a-priori inner semicontinuity of $\Gamma$. Here, we follow the ideas used for the proof of [36, Theorem 2].

Theorem 3.1 Let (A1) and (A2) hold. Then the following statements are equivalent.

(a) The mapping $\Gamma$ is $R$-regular at $(\bar{x}, \bar{y})$ w.r.t. $\operatorname{dom} \Gamma$.

(b) There exists a constant $M>0$ such that for each sequences $\left\{x^{k}\right\}_{k \in \mathbb{N}} \subset \operatorname{dom} \Gamma$, $\left\{v^{k}\right\}_{k \in \mathbb{N}} \subset \mathbb{R}^{m}$, and $\left\{y^{k}\right\}_{k \in \mathbb{N}} \subset \mathbb{R}^{m}$ satisfying $x^{k} \rightarrow \bar{x}, v^{k} \rightarrow \bar{y}$, and $v^{k} \notin \Gamma\left(x^{k}\right)$ as well as $y^{k} \in \Pi\left(v^{k}, \Gamma\left(x^{k}\right)\right)$ for all $k \in \mathbb{N}$, the set $\Lambda_{v^{k}}^{M}\left(x^{k}, y^{k}\right)$ is nonempty for sufficiently large $k \in \mathbb{N}$.

Proof We show both implications separately.

(a) $\Longrightarrow$ (b): Let $\Gamma$ be R-regular at $(\bar{x}, \bar{y})$ w.r.t. $\operatorname{dom} \Gamma$. Then we find $\kappa>0$ and $\gamma, \delta>0$ such that

$$
\begin{aligned}
& \forall x \in \mathbb{U}_{\gamma}(\bar{x}) \cap \operatorname{dom} \Gamma \forall y \in \mathbb{U}_{\delta}(\bar{y}): \\
& \quad \operatorname{dist}(y, \Gamma(x)) \leq \kappa \max \left\{0, \max \left\{h_{i}(x, y) \mid i \in I\right\}, \max \left\{\left|h_{i}(x, y)\right| \mid i \in J\right\}\right\}
\end{aligned}
$$

holds. Furthermore, let $\left\{x^{k}\right\}_{k \in \mathbb{N}} \subset \operatorname{dom} \Gamma,\left\{v^{k}\right\}_{k \in \mathbb{N}} \subset \mathbb{R}^{m}$, and $\left\{y^{k}\right\}_{k \in \mathbb{N}} \subset \mathbb{R}^{m}$ be sequences which satisfy the requirements in (b). We first show $y^{k} \rightarrow \bar{y}$. Indeed, we have

$$
\left\|y^{k}-\bar{y}\right\| \leq\left\|y^{k}-v^{k}\right\|+\left\|v^{k}-\bar{y}\right\|=\operatorname{dist}\left(v^{k}, \Gamma\left(x^{k}\right)\right)+\left\|v^{k}-\bar{y}\right\|,
$$

and the term on the right tends to zero as $k \rightarrow \infty$ by R-regularity of $\Gamma$ at $(\bar{x}, \bar{y})$ and continuity of $h_{1}, \ldots, h_{p}$ at $(\bar{x}, \bar{y})$.

Fix $k \in \mathbb{N}$ and define mappings $\Phi_{k}, \Psi_{k}: \mathbb{R}^{m} \rightarrow \mathbb{R}$ by means of

$$
\forall w \in \mathbb{R}^{m}: \quad \Phi_{k}(w):=\left\|w-v^{k}\right\|, \quad \Psi_{k}(w):=\Phi_{k}(w)+2 \operatorname{dist}\left(w, \Gamma\left(x^{k}\right)\right) .
$$


Observing that $\Phi_{k}$ is globally Lipschitz continuous with Lipschitz modulus 1 while $\Gamma\left(x^{k}\right)$ is nonempty and closed, Clarke's principle of exact penalization, see [11, Proposition 2.4.3], implies that $y^{k}$ is a global minimizer of $\Psi_{k}$.

For sufficiently large $k \in \mathbb{N}$, we have $x^{k} \in \mathbb{U}_{\gamma}(\bar{x})$ and $y^{k} \in \mathbb{U}_{\delta / 2}(\bar{y})$. Consider such $k \in \mathbb{N}$ and an arbitrary vector $w \in \mathbb{U}_{\delta / 2}\left(y^{k}\right)$. Then the above considerations and (3.2) yield the estimate

$$
\begin{aligned}
\Phi_{k}\left(y^{k}\right) & =\Psi_{k}\left(y^{k}\right) \leq \Psi_{k}(w)=\Phi_{k}(w)+2 \operatorname{dist}\left(w, \Gamma\left(x^{k}\right)\right) \\
\leq & \Phi_{k}(w)+2 \kappa \max \left\{0, \max \left\{h_{i}\left(x^{k}, w\right) \mid i \in I\right\}, \max \left\{\left|h_{i}\left(x^{k}, w\right)\right| \mid i \in J\right\}\right\} \\
& =\max \left\{\begin{array}{l|l}
\Phi_{k}(w)+\sum_{i=1}^{p} \lambda_{i} h_{i}\left(x^{k}, w\right) & \begin{array}{l}
\forall i \in I: \lambda_{i} \geq 0, \lambda_{i} \min \left\{0, h_{i}\left(x^{k}, w\right)\right\}=0 \\
\sum_{i=1}^{p}\left|\lambda_{i}\right| \leq 2 \kappa
\end{array}
\end{array}\right\} .
\end{aligned}
$$

Using the function $\mathcal{L}_{k}: \mathbb{U}_{\delta / 2}\left(y^{k}\right) \times \mathbb{R}^{p} \rightarrow \mathbb{R}$ and the set $\widetilde{\Lambda}_{k}(w)$ given by

$$
\begin{aligned}
\mathcal{L}_{k}(w, \lambda) & :=\Phi_{k}(w)+\sum_{i=1}^{p} \lambda_{i} h_{i}\left(x^{k}, w\right) \\
\widetilde{\Lambda}^{k}(w) & :=\left\{\lambda \in \mathbb{R}^{p}\left|\sum_{i=1}^{p}\right| \lambda_{i} \mid \leq 2 \kappa, \forall i \in I: \lambda_{i} \geq 0, \lambda_{i} \min \left\{0, h_{i}\left(x^{k}, w\right)\right\}=0\right\}
\end{aligned}
$$

for all $w \in \mathbb{U}_{\delta / 2}\left(y^{k}\right)$ and $\lambda \in \mathbb{R}^{p}$, we have

$$
\forall w \in \mathbb{U}_{\delta / 2}\left(y^{k}\right): \quad \Phi_{k}\left(y^{k}\right) \leq \max \left\{\mathcal{L}_{k}(w, \lambda) \mid \lambda \in \widetilde{\Lambda}^{k}(w)\right\} .
$$

By continuity of the functions $h_{i}\left(x^{k}, \cdot\right), i=1, \ldots, \ell$, the inclusion $\widetilde{\Lambda}^{k}(w) \subset \widetilde{\Lambda}^{k}\left(y^{k}\right)$ holds for all $w \in \mathbb{U}_{\delta / 2}\left(y^{k}\right)$ close enough to $y^{k}$ which is why we find $\delta_{k}^{\prime} \in(0, \delta / 2]$ such that

$$
\forall w \in \mathbb{U}_{\delta_{k}^{\prime}}\left(y^{k}\right): \quad \Phi_{k}\left(y^{k}\right) \leq \max \left\{\mathcal{L}_{k}(w, \lambda) \mid \lambda \in \widetilde{\Lambda}^{k}\left(y^{k}\right)\right\} .
$$

Defining $\mathcal{Q}_{k}: \mathbb{U}_{\delta_{k}^{\prime}}\left(y^{k}\right) \rightarrow \mathbb{R}$ by means of

$$
\forall w \in \mathbb{U}_{\delta_{k}^{\prime}}\left(y^{k}\right): \quad \mathcal{Q}_{k}(w):=\max \left\{\mathcal{L}_{k}(w, \lambda) \mid \lambda \in \widetilde{\Lambda}^{k}\left(y^{k}\right)\right\},
$$

we obtain $\Phi_{k}\left(y^{k}\right) \leq \mathcal{Q}_{k}(w)$ for all $w \in \mathbb{U}_{\delta_{k}^{\prime}}\left(y^{k}\right)$ from (3.3). Furthermore, $\Phi_{k}\left(y^{k}\right)=$ $\mathcal{Q}_{k}\left(y^{k}\right)$ holds which is why $y^{k}$ is a global minimizer of $\mathcal{Q}_{k}$. For sufficiently large $k \in \mathbb{N}$, $\mathcal{L}_{k}$ is continuously differentiable at $y^{k}$. Noting that $\tilde{\Lambda}^{k}\left(y^{k}\right)$ is a compact polyhedron, $\mathcal{Q}_{k}$ is directionally differentiable at $y^{k}$, and the directional derivative can be approximated from above by means of

$$
\forall d \in \mathbb{R}^{m}: \quad \mathcal{Q}_{k}^{\prime}\left(y^{k}, d\right) \leq \max \left\{\nabla_{y} \mathcal{L}_{k}\left(y^{k}, \lambda\right)^{\top} d \mid \lambda \in \widetilde{\Lambda}^{k}\left(y^{k}\right)\right\}
$$

which follows from Danskin's theorem, see [7, Proposition B.25], due to validity of (A1). Recalling that $y^{k}$ is a global minimizer of $\mathcal{Q}_{k}$, we have $\mathcal{Q}_{k}^{\prime}\left(y^{k}, d\right) \geq 0$ for all $d \in \mathbb{R}^{m}$. Defining a polytope $P \subset \mathbb{R}^{m}$ by means of

$$
P:=\left\{\nabla_{y} \mathcal{L}_{k}\left(y^{k}, \lambda\right) \mid \lambda \in \widetilde{\Lambda}^{k}\left(y^{k}\right)\right\},
$$

we find $\max \left\{\xi^{\top} d \mid \xi \in P\right\} \geq 0$ for all $d \in \mathbb{R}^{m}$. This yields $0 \in P$. By definition of $P, \mathcal{L}_{k}$, and $\widetilde{\Lambda}^{k}, \Lambda_{\nu^{k}}^{2 \kappa}\left(x^{k}, y^{k}\right) \neq \varnothing$ follows. Since the above arguments apply to all sufficiently large $k \in \mathbb{N}$, (b) holds.

(b) $\Longrightarrow$ (a): Let (b) hold and assume that $\Gamma$ is not R-regular at $(\bar{x}, \bar{y})$ w.r.t. $\operatorname{dom} \Gamma$. Then we find sequences $\left\{x^{k}\right\}_{k \in \mathbb{N}} \subset \operatorname{dom} \Gamma$ and $\left\{v^{k}\right\}_{k \in \mathbb{N}} \subset \mathbb{R}^{m}$ such that $x^{k} \rightarrow \bar{x}, v^{k} \rightarrow \bar{y}$, and

$$
\operatorname{dist}\left(v^{k}, \Gamma\left(x^{k}\right)\right) \geq k \max \left\{0, \max \left\{h_{i}\left(x^{k}, v^{k}\right) \mid i \in I\right\}, \max \left\{\left|h_{i}\left(x^{k}, v^{k}\right)\right| \mid i \in J\right\}\right\}
$$


as well as $v^{k} \notin \Gamma\left(x^{k}\right)$ hold for all $k \in \mathbb{N}$. For each $k \in \mathbb{N}$, we fix $y^{k} \in \Pi\left(v^{k}, \Gamma\left(x^{k}\right)\right)$. Due to validity of (b), the set $\Lambda_{v^{k}}^{M}\left(x^{k}, y^{k}\right)$ is nonempty for sufficiently large $k \in \mathbb{N}$. By means of (A1) and (A2), Lemma 3.1 yields a contradiction since (3.1) and (3.4) are incongruous.

\subsection{R-Regularity Under RCPLD}

In this section, we want to exploit the sequential characterization of R-regularity obtained in Theorem 3.1 in order to show that validity of RCPLD is a sufficient criterion for R-regularity in the presence of (A1) and (A2). This generalizes [6, Theorem 4.2] and [36, Theorem 4] where a-priori inner semicontinuity of $\Gamma$ at the reference point as well as RCRCQ were the necessary ingredients to come up with a related result in the absence of (A1) and (A2).

Theorem 3.2 Let (A1) and (A2) hold. Suppose that RCPLD holds at each point from the set $\{\bar{x}\} \times \Gamma(\bar{x})$ w.r.t. $\operatorname{dom} \Gamma$. Then $\Gamma$ is R-regular at each point from $\{\bar{x}\} \times \Gamma(\bar{x})$ w.r.t. $\operatorname{dom} \Gamma$.

Proof Suppose that there exists $\tilde{y} \in \Gamma(\bar{x})$ such that $\Gamma$ is not R-regular at $(\bar{x}, \tilde{y})$ w.r.t. $\operatorname{dom} \Gamma$. Due to Theorem 3.1, this shows that for each $\sigma \in \mathbb{N}$, there exist sequences $\left\{x_{\sigma}^{k}\right\}_{k \in \mathbb{N}} \subset$ $\operatorname{dom} \Gamma,\left\{v_{\sigma}^{k}\right\}_{k \in \mathbb{N}} \subset \mathbb{R}^{m}$, and $\left\{y_{\sigma}^{k}\right\}_{k \in \mathbb{N}} \subset \mathbb{R}^{m}$ satisfying $x_{\sigma}^{k} \rightarrow \bar{x}, v_{\sigma}^{k} \rightarrow \tilde{y}, v_{\sigma}^{k} \notin \Gamma\left(x_{\sigma}^{k}\right)$ as well as $y_{\sigma}^{k} \in \Pi\left(v_{\sigma}^{k}, \Gamma\left(x_{\sigma}^{k}\right)\right)$ for all $k \in \mathbb{N}$, and $\Lambda_{v_{\sigma}^{k_{s}}}^{\sigma}\left(x_{\sigma}^{k_{s}}, y_{\sigma}^{k_{s}}\right)=\varnothing$ for all $s \in \mathbb{N}$, i.e., the latter holds at least on a subsequence. Performing a standard diagonal sequence argument, we, thus, find sequences $\left\{x^{\sigma}\right\}_{\sigma \in \mathbb{N}} \subset \operatorname{dom} \Gamma,\left\{v^{\sigma}\right\}_{\sigma \in \mathbb{N}} \subset \mathbb{R}^{m}$, and $\left\{y^{\sigma}\right\}_{\sigma \in \mathbb{N}}$ satisfying $x^{\sigma} \rightarrow \bar{x}$, $v^{\sigma} \rightarrow \tilde{y}$, as well as $v^{\sigma} \notin \Gamma\left(x^{\sigma}\right), y^{\sigma} \in \Pi\left(v^{\sigma}, \Gamma\left(x^{\sigma}\right)\right)$, and $\Lambda_{v^{\sigma}}^{\sigma}\left(x^{\sigma}, y^{\sigma}\right)=\varnothing$ for all $\sigma \in \mathbb{N}$.

Invoking (A2) and the continuity of $h_{1}, \ldots, h_{p}$ at each point from $\{\bar{x}\} \times \Gamma(\bar{x})$, we obtain that for each $\varepsilon>0$, there is a $\delta>0$ such that $\Gamma(x) \subset \Gamma(\bar{x})+\varepsilon \mathbb{B}$ holds for all $x \in$ $\mathbb{U}_{\delta}(\bar{x})$ since $\Gamma$ is upper semicontinuous at $\bar{x}$, see [42, Theorem 5.19] as well. Thus, recalling that RCPLD is locally stable, it needs to hold at the points $\left(x^{\sigma}, y^{\sigma}\right)$ for sufficiently large $\sigma \in \mathbb{N}$. Exploiting the fact that RCPLD is, actually, a constraint qualification, this implies $\Lambda_{v^{\sigma}}\left(x^{\sigma}, y^{\sigma}\right) \neq \varnothing$. Since we have $\Lambda_{v^{\sigma}}^{\sigma}\left(x^{\sigma}, y^{\sigma}\right)=\varnothing$ from above, we conclude that each sequence $\left\{\mu^{\sigma}\right\}_{\sigma \in \mathbb{N}}$ with $\mu^{\sigma} \in \Lambda_{\nu^{\sigma}}\left(x^{\sigma}, y^{\sigma}\right)$ for all $\sigma \in \mathbb{N}$ satisfies $\left\|\mu^{\sigma}\right\| \rightarrow \infty$ as $\sigma \rightarrow \infty$. Choose such a sequence. Recall that this means

$$
\begin{aligned}
& 0=\frac{y^{\sigma}-v^{\sigma}}{\left\|y^{\sigma}-v^{\sigma}\right\|}+\sum_{i=1}^{p} \mu_{i}^{\sigma} \nabla_{y} h_{i}\left(x^{\sigma}, y^{\sigma}\right), \\
& \forall i \in I\left(x^{\sigma}, y^{\sigma}\right): \mu_{i}^{\sigma} \geq 0 \\
& \forall i \in I \backslash I\left(x^{\sigma}, y^{\sigma}\right): \mu_{i}^{\sigma}=0
\end{aligned}
$$

for all $\sigma \in \mathbb{N}$ sufficiently large.

Clearly, (A2) guarantees that $\left\{y^{\sigma}\right\}_{\sigma \in \mathbb{N}}$ is locally bounded and, thus, converges along a subsequence (without relabeling) to some $\bar{y} \in \Gamma(\bar{x})$ by continuity of $h_{1}, \ldots, h_{p}$ at each point from $\{\bar{x}\} \times \Gamma(\bar{x})$. Since RCPLD holds at $(\bar{x}, \bar{y})$ w.r.t. $\operatorname{dom} \Gamma$, we find a neighborhood $U$ of this point as well as an index set $S \subset J$ satisfying the requirements (i), (ii), and (iii) from part (d) of Definition 2.1. Particularly, the family $\left(\nabla_{y} h_{i}(x, y)\right)_{i \in S}$ needs to be linearly independent while the vectors from $\left(\nabla_{y} h_{i}(x, y)\right)_{i \in J \backslash S}$ need to be linearly dependent on the family $\left(\nabla_{y} h_{i}(x, y)\right)_{i \in S}$ for all $(x, y) \in U \cap\left(\operatorname{dom} \Gamma \times \mathbb{R}^{m}\right)$. For sufficiently large $\sigma \in \mathbb{N}$, $\left(x^{\sigma}, y^{\sigma}\right) \in U \cap\left(\operatorname{dom} \Gamma \times \mathbb{R}^{m}\right)$ holds true. The above arguments lead to the existence of $\bar{\mu}_{i}^{\sigma}$, $i \in J$, such that

$$
\sum_{i \in J} \mu_{i}^{\sigma} \nabla_{y} h_{i}\left(x^{\sigma}, y^{\sigma}\right)=\sum_{i \in S} \bar{\mu}_{i}^{\sigma} \nabla_{y} h_{i}\left(x^{\sigma}, y^{\sigma}\right),
$$


holds for sufficiently large $\sigma \in \mathbb{N}$ where, additionally, the family $\left(\nabla_{y} h_{i}\left(x^{\sigma}, y^{\sigma}\right)\right)_{i \in S}$ is linearly independent. Now, (3.6a) allows to rewrite (3.5a) as

$$
0=\frac{y^{\sigma}-v^{\sigma}}{\left\|y^{\sigma}-v^{\sigma}\right\|}+\sum_{i \in I} \mu_{i}^{\sigma} \nabla_{y} h_{i}\left(x^{\sigma}, y^{\sigma}\right)+\sum_{i \in S} \bar{\mu}_{i}^{\sigma} \nabla_{y} h_{i}\left(x^{\sigma}, y^{\sigma}\right)
$$

for sufficiently large $\sigma \in \mathbb{N}$. Observing that there are only finitely many subsets of $I$, we may pass to a subsequence (without relabeling) in order to guarantee $I\left(x^{\sigma}, y^{\sigma}\right)=\mathfrak{I}$ for all $\sigma \in \mathbb{N}$ and some set $\mathfrak{I} \subset I$. Now, we apply Lemma 2.1 to the situation at hand. Thus, for each sufficiently large $\sigma \in \mathbb{N}$, we find a set $I^{\sigma} \subset \mathfrak{I}$ as well as reals $\bar{\lambda}_{i}^{\sigma}, i \in I^{\sigma} \cup S$, satisfying $\bar{\lambda}_{i}^{\sigma}>0$ for all $i \in I^{\sigma}$, such that the family $\left(\nabla_{y} h_{i}\left(x^{\sigma}, y^{\sigma}\right)\right)_{i \in I^{\sigma} \cup S}$ is linearly independent while

$$
0=\frac{y^{\sigma}-v^{\sigma}}{\left\|y^{\sigma}-v^{\sigma}\right\|}+\sum_{i \in I^{\sigma} \cup S} \bar{\lambda}_{i}^{\sigma} \nabla_{y} h_{i}\left(x^{\sigma}, y^{\sigma}\right)
$$

holds for all $\sigma \in \mathbb{N}$. By passing once more to a subsequence (without relabeling), we may ensure that $I^{\sigma}=\mathcal{I}$ holds for all $\sigma \in \mathbb{N}$ and some index set $\mathcal{I} \subset I$. Let us set $\bar{\lambda}_{i}^{\sigma}:=0$ for all $i \in(I \backslash \mathcal{I}) \cup(J \backslash S)$ in order to rewrite the above equation as

$$
0=\frac{y^{\sigma}-v^{\sigma}}{\left\|y^{\sigma}-v^{\sigma}\right\|}+\sum_{i=1}^{p} \bar{\lambda}_{i}^{\sigma} \nabla_{y} h_{i}\left(x^{\sigma}, y^{\sigma}\right) .
$$

Thus, we have shown $\bar{\lambda}^{\sigma} \in \Lambda_{v^{\sigma}}\left(x^{\sigma}, y^{\sigma}\right)$. The above arguments show $\left\|\bar{\lambda}^{\sigma}\right\| \rightarrow \infty$ as $\sigma \rightarrow \infty$. Consequently, dividing (3.7) by $\left\|\bar{\lambda}^{\sigma}\right\|$ and taking the limit $\sigma \rightarrow \infty$, we infer

$$
\begin{aligned}
& 0=\sum_{i=1}^{p} \bar{\lambda}_{i} \nabla_{y} h_{i}(\bar{x}, \bar{y}), \\
& \forall i \in \mathcal{I}: \bar{\lambda}_{i} \geq 0, \\
& \forall i \in(I \backslash \mathcal{I}) \cup(J \backslash S): \bar{\lambda}_{i}=0
\end{aligned}
$$

for some non-vanishing multiplier $\bar{\lambda} \in \mathbb{R}^{p}$ by the assumed continuity of the derivatives $\nabla_{y} h_{1}, \ldots, \nabla_{y} h_{p}$ at $(\bar{x}, \bar{y})$. Thus, the pair of families $\left(\left(\nabla_{y} h_{i}(\bar{x}, \bar{y})\right)_{i \in \mathcal{I}},\left(\nabla_{y} h_{i}(\bar{x}, \bar{y})\right)_{i \in S}\right)$ is positive-linearly dependent. On the other hand, we have already shown above that the families $\left(\nabla_{y} h_{i}\left(x^{\sigma}, y^{\sigma}\right)\right)_{i \in \mathcal{I} \cup S}$ are linearly independent. This, however, contradicts the validity of RCPLD at $(\bar{x}, \bar{y})$ and, thus, completes the proof.

As a consequence of the above theorem and Lemma 2.2, we obtain the following corollary.

Corollary 3.1 Let the assumptions of Theorem 3.2 hold. Then $\Gamma$ is lower semicontinuous w.r.t. $\operatorname{dom} \Gamma$ at $\bar{x}$.

Inspecting the proofs of Lemma 3.1 as well as Theorems 3.1 and 3.2, one can check that continuity of all involved functions w.r.t. the set $\operatorname{dom} \Gamma \times \mathbb{R}^{m}$ is actually enough in order to proceed. A remark, which provides another slight generalization of our setting, is presented below.

Remark 3.1 Observe that the proofs of Lemma 3.1 as well as Theorems 3.1 and 3.2 remain true in the following setting which is slightly more general than the one of Assumption 2.1: For each $i \in\{1, \ldots, p\}$, there exist functions $g_{i}: \mathbb{R}^{n} \times \mathbb{R}^{m} \rightarrow \mathbb{R}$ and $t_{i}: \mathbb{R}^{n} \rightarrow \overline{\mathbb{R}}$ such that $h_{i}(x, y)=g_{i}(x, y)+t_{i}(x)$ holds true for all $(x, y) \in \mathbb{R}^{n} \times \mathbb{R}^{m}$. Furthermore, $g_{i}$ is continuous as well as continuously differentiable w.r.t. $y$ in a neighborhood of $\{\bar{x}\} \times \Gamma(\bar{x})$. Finally, we have $\left|t_{i}(x)\right|<\infty$ for all $x \in \operatorname{dom} \Gamma$ from a neighborhood of $\bar{x}$ and $t_{i}$ is continuous at $\bar{x}$. 
Observe that the assertion of Theorem 3.2 is essentially different from the one of [6, Theorem 4.2]. In [6], the authors claimed validity of inner semicontinuity and RCRCQ at one point from the graph of $\Gamma$ in order to obtain R-regularity at the reference point. Here, however, we postulate (A1) and assume validity of RCPLD at all points from $\{\bar{x}\} \times \Gamma(\bar{x})$ in order to deduce R-regularity of $\Gamma$ at all these points. Thus, in this setting, one may interpret the statement of Theorem 3.2 as a sufficient condition for lower semicontinuity of $\Gamma$ as well, see Corollary 3.1. Observe that we cannot modify the statement of Theorem 3.2 in such a way that assuming validity of $\operatorname{RCPLD}$ at one reference point $(\bar{x}, \bar{y}) \in \operatorname{gph} \Gamma$ ensures R-regularity of $\Gamma$ at the same point without adding inner semicontinuity of $\Gamma$ at $(\bar{x}, \bar{y})$ while relying on the provided proof. However, we obtain the following result which generalizes [6, Theorem 4.2].

Theorem 3.3 Assume that $\Gamma$ is inner semicontinuous at $(\bar{x}, \bar{y})$ w.r.t. $\operatorname{dom} \Gamma$ and let $R C P L D$ hold at this point w.r.t. $\operatorname{dom} \Gamma$. Then $\Gamma$ is $R$-regular at $(\bar{x}, \bar{y})$ w.r.t. $\operatorname{dom} \Gamma$.

Proof We follow the lines of the proof of Theorem 3.2 while respecting the following changes: First, the role of $\tilde{y}$ is played by $\bar{y}$. Second, inner semicontinuity of $\Gamma$ at $(\bar{x}, \bar{y})$ w.r.t. $\operatorname{dom} \Gamma$ ensures validity of the sequential characterization of R-regularity from Theorem 3.1 in the absence of (A1) and (A2), see [6, Theorem 3.2]. Third, inner semicontinuity of $\Gamma$ at $(\bar{x}, \bar{y})$ w.r.t. dom $\Gamma$ can be used to infer the convergence $y^{\sigma} \rightarrow \bar{y}$ without presuming validity of (A2). Fourth, the relation $\Lambda_{v^{\sigma}}\left(x^{\sigma}, y^{\sigma}\right) \neq \varnothing$ follows for sufficiently large $\sigma \in \mathbb{N}$ directly from local stability of RCPLD.

Let us note that all the assumptions of Theorem 3.3 hold whenever MFCQ is valid at $(\bar{x}, \bar{y}) \in \operatorname{gph} \Gamma$. In this case, $\Gamma$ is inner semicontinuous at $(\bar{x}, \bar{y})$, see [22, Section III] for a demonstration, which particularly yields that $\bar{x}$ is an interior point of $\operatorname{dom} \Gamma$, i.e., $\Gamma$ is R-regular at $(\bar{x}, \bar{y})$ in this case. Note that, on the other hand, validity of MFCQ at $(\bar{x}, \bar{y})$ guarantees R-regularity of $\Gamma$ at this point by means of e.g. [8, Theorem 3.2]. Due to Lemma 2.2, this also shows that $\Gamma$ is inner semicontinuous at $(\bar{x}, \bar{y})$ and, thus, that $\bar{x}$ belongs to the interior of $\operatorname{dom} \Gamma$.

Let us point out that in case where $\Gamma$ does not depend on the parameter $x$, Theorem 3.3 provides a sufficient condition for the presence of an error bound at some reference point of a nonlinear constraint system. For a similar result under slightly stronger assumptions, we refer the interested reader to [1, Theorem 7]. Furthermore, we would like to mention [10, Theorem 4.2] where this result has been obtained in the context of mathematical problems with complementarity constraints.

Using the popular tools of directional limiting variational analysis, the authors in [24] struck a completely different path in order to derive first- and second-order sufficient conditions for the R-regularity of $\Gamma$ which are also weaker than MFCQ. However, in order to obtain a first-order sufficient condition in terms of initial problem data from [24, Theorem 3.5], differentiability of the functions $h_{1}, \ldots, h_{p}$ w.r.t. the parameter as well as injectivity of the associated derivative seems to be necessary, and this is far beyond the regularity which was necessary in order to derive Theorems 3.2 and 3.3.

The upcoming example, which closes this section, shows that the statements of Theorems 3.2 and 3.3 do not need to hold in the absence of the convexity assumption (A1) or the inner semicontinuity of $\Gamma$ at the reference point, respectively.

Example 3.1 We consider the mapping $\Gamma: \mathbb{R} \rightrightarrows \mathbb{R}$ given by

$$
\forall x \in \mathbb{R}: \quad \Gamma(x):=\left\{y \in \mathbb{R} \mid x-y \leq 0, y-y^{2} \leq 0, y-1 \leq 0\right\} .
$$


A simple calculation reveals

$$
\forall x \in \mathbb{R}: \quad \Gamma(x)= \begin{cases}{[x, 0] \cup\{1\}} & x \in(-\infty, 0], \\ \{1\} & x \in(0,1], \\ \varnothing & x \in(1, \infty) .\end{cases}
$$

We study the point $\bar{x}:=0$ as well as the associated images $\bar{y}:=0$ and $\tilde{y}:=1$ in $\Gamma(\bar{x})$. Note that $\Gamma$ is inner semicontinuous at $(\bar{x}, \tilde{y})$ but not at $(\bar{x}, \bar{y})$. Thus, $\Gamma$ cannot be R-regular at $(\bar{x}, \bar{y})$ due to Lemma 2.2 .

Observe that the family $(-1,1-2 y)$ is positive-linearly dependent around $\bar{y}$ while the family $(1-2 y, 1)$ is positive-linearly dependent around $\tilde{y}$. Thus, RCPLD is valid at $(\bar{x}, \bar{y})$ and $(\bar{x}, \tilde{y})$, respectively. This shows that the statement of Theorem 3.2 does not generally hold in the absence of (A1) while the assertion of Theorem 3.3 is not generally true if $\Gamma$ is not inner semicontinuous at the reference point.

\section{Applications}

\subsection{Parametric Optimization}

For a function $f: \mathbb{R}^{n} \times \mathbb{R}^{m} \rightarrow \mathbb{R}$, we investigate the parametric optimization problem

$$
\min _{y}\{f(x, y) \mid y \in \Gamma(x)\}
$$

where $\Gamma: \mathbb{R}^{n} \rightrightarrows \mathbb{R}^{m}$ is the set-valued mapping given in (1.1). Associated with the problem $(\mathrm{P}(x))$ are the solution mapping $S: \mathbb{R}^{n} \rightrightarrows \mathbb{R}^{m}$ given by

$$
\forall x \in \mathbb{R}^{n}: \quad S(x):=\underset{y}{\operatorname{argmin}}\{f(x, y) \mid y \in \Gamma(x)\}
$$

as well as the optimal value (or marginal) function $\varphi: \mathbb{R}^{n} \rightarrow \overline{\mathbb{R}}$ defined via

$$
\forall x \in \mathbb{R}^{n}: \quad \varphi(x):=\inf _{y}\{f(x, y) \mid y \in \Gamma(x)\} .
$$

Clearly, we have the relation

$$
\forall x \in \mathbb{R}^{n}: \quad S(x)=\{y \in \Gamma(x) \mid f(x, y) \leq \varphi(x)\}
$$

which is why $S$ can be interpreted as a solution mapping associated with a parametric system of nonlinear inequalities and equations. It is well known that under comparatively weak assumptions, the optimal value function $\varphi$ is continuous at a given reference point, see e.g. [4]. Keeping Remark 3.1 in mind, we are thus in position to apply the theory from Section 3 to this representation of $S$ in order to infer its R-regularity at a given reference point under suitable assumptions. This way, we also obtain new sufficient criteria for the presence of the Aubin property of $S$ or its inner semicontinuity at a given reference point. For the sake of brevity and consistency, we define $h_{0}: \mathbb{R}^{n} \times \mathbb{R}^{m} \rightarrow \overline{\mathbb{R}}$ by means of

$$
\forall x \in \mathbb{R}^{n} \forall y \in \mathbb{R}^{m}: \quad h_{0}(x, y):=f(x, y)-\varphi(x)
$$

and emphasize that $S$ possesses the representation

$$
\forall x \in \mathbb{R}^{n}: \quad S(x)=\left\{\begin{array}{l|l}
y \in \mathbb{R}^{m} & \begin{array}{l}
h_{i}(x, y) \leq 0 \quad i \in I \cup\{0\} \\
h_{i}(x, y)=0 \quad i \in J
\end{array}
\end{array}\right\} .
$$

This representation of $S$ can be addressed with the theory from Section 3. In this section, we need to refer to the parametric constraint systems induced by $\Gamma$ and $S$, individually. In this regard, we will exploit the notation $\mathrm{RCPLD}_{\Gamma}$ and $\mathrm{RCPLD}_{S}$ in order to avoid any confusion. 
Let us emphasize that, if not stated otherwise, we will include the constraint function $h_{0}$ as an inequality constraint when considering $S$, i.e., we exploit the representation of $S$ from (4.1) in most of the cases. However, it is also possible to incorporate $h_{0}$ as an equality constraint.

Remark 4.1 We also have the representation

$$
\forall x \in \mathbb{R}^{n}: \quad S(x)=\left\{\begin{array}{l|l}
y \in \mathbb{R}^{m} & \begin{array}{l}
h_{i}(x, y) \leq 0 \quad i \in I \\
h_{i}(x, y)=0 \quad i \in J \cup\{0\}
\end{array}
\end{array}\right\},
$$

and, in some situations, it might be beneficial to apply the theory of Section 3 to this representation of $S$ instead of the one from (4.1).

We postulate the following standing assumption throughout the section.

Assumption 4.1 The functions $f$ and $h_{1}, \ldots, h_{p}$ are continuously differentiable.

Note that by continuity of $h_{1}, \ldots, h_{p}$, we already know that gph $\Gamma$ is closed. Particularly, the image sets of $\Gamma$ are closed. By continuity of $f$, we even know that the image sets of $S$ are closed.

Finally, we will exploit the following modified version of (A1) in some situations:

(A1') For each $x \in \mathbb{R}^{n}$, the functions $f(x, \cdot): \mathbb{R}^{m} \rightarrow \mathbb{R}$ and $h_{i}(x, \cdot): \mathbb{R}^{m} \rightarrow \mathbb{R}, i \in I$, are convex while the functions $h_{i}(x, \cdot): \mathbb{R}^{m} \rightarrow \mathbb{R}, i \in J$, are affine.

We note that (A1') is the counterpart of (A1) which addresses the representation of $S$ from (4.1). In case where one aims to exploit the representation of $S$ from Remark 4.1, the convexity of $f(x, \cdot): \mathbb{R}^{m} \rightarrow \mathbb{R}$ for each $x \in \mathbb{R}^{n}$ has to be replaced by the property of this mapping to be affine.

\subsubsection{Continuity Properties of Marginal Functions}

In the subsequent lemma, we collect some results regarding the continuity properties of the function $\varphi$. The proof is stated for the reader's convenience.

Lemma 4.1 Fix a point $\bar{x} \in \operatorname{dom} \Gamma$ where (A2) is valid. Then the following assertions hold.

(a) The function $\varphi$ is lower semicontinuous at $\bar{x}$.

(b) Assume that there exists $\bar{y} \in \Gamma(\bar{x})$ such that $\Gamma$ is inner semicontinuous at $(\bar{x}, \bar{y})$ w.r.t. dom $\Gamma$. Then $\varphi$ is continuous at $\bar{x}$ w.r.t. dom $\Gamma$.

(c) Assume that $\Gamma$ possesses the Aubin property at each point from $\{\bar{x}\} \times S(\bar{x})$. Then $\varphi$ is locally Lipschitz continuous at $\bar{x}$.

(d) Assume that there exists $\bar{y} \in S(\bar{x})$ such that $\Gamma$ possesses the Aubin property at $(\bar{x}, \bar{y})$ while $S$ is inner semicontinuous at this point. Then $\varphi$ is locally Lipschitz continuous at $\bar{x}$.

Proof (a) By continuity of the functions $h_{1}, \ldots, h_{p}$ and validity of (A2), we obtain upper semicontinuity of $\Gamma$ at $\bar{x}$. Thus, the desired assertion can be distilled from [4, Theorem 4.2.1] since $f$ is continuous.

(b) Consulting the proof of [4, Theorem 4.2.1], inner semicontinuity of $\Gamma$ at $(\bar{x}, \bar{y})$ is enough to guarantee that $\varphi$ is upper semicontinuous at $\bar{x}$ since $f$ is continuous. Combining this with (a), the desired result follows. 
(c) Due to validity of (A2), the solution mapping $S$ is locally bounded at $\bar{x}$ as well. Particularly, $S$ possesses bounded images in a neighborhood of $\bar{x}$. Due to $\bar{x} \in \operatorname{dom} \Gamma$, we have $\Gamma(\bar{x}) \neq \varnothing$ and, thus, $S(\bar{x}) \neq \varnothing$ by Weierstrass' theorem. Since $\Gamma$ possesses the Aubin property at each point from $\{\bar{x}\} \times S(\bar{x}), \Gamma$ is inner semicontinuous at each point $(\bar{x}, y) \in \operatorname{gph} S$ and, thus, possesses nonempty image sets in a neighborhood of $\bar{x}$. Thus, we deduce that $S$ possesses bounded and nonempty image sets in a neighborhood of $\bar{x}$. Furthermore, $\varphi$ is lower semicontinuous at $\bar{x}$ by (a). Thus, the statement follows from [38, Theorem 5.3(ii)].

(d) This follows directly from [38, Theorem 5.3(i)] while observing that $\varphi$ is continuous at $\bar{x}$ by inner semicontinuity of $S$ at $(\bar{x}, \bar{y})$ and continuity of $f$.

We would like to mention that statement (d) of Lemma 4.1 holds even true in the absence of (A2) since the latter has not been used in the proof.

As a corollary of Theorems 3.2 and 3.3 as well as Lemma 4.1, we obtain the following result as a consequence of the local Lipschitz continuity of the functions $h_{1}, \ldots, h_{p}$ since the latter implies that R-regularity of $\Gamma$ at some point of its graph already guarantees validity of the Aubin property there.

Corollary 4.1 Fix some point $\bar{x} \in \operatorname{dom} \Gamma$. Let one of the following additional assumptions be valid.

(a) Let (A1) and (A2) hold. Furthermore, let $R C P L D_{\Gamma}$ hold at each point from $\{\bar{x}\} \times \Gamma(\bar{x})$ and assume that $\bar{x}$ is an interior point of $\operatorname{dom} \Gamma$.

(b) Let $\bar{y} \in S(\bar{x})$ be chosen such that $S$ is inner semicontinuous at $(\bar{x}, \bar{y})$ while $R C P L D_{\Gamma}$ holds at this point.

Then $\varphi$ is locally Lipschitz continuous at $\bar{x}$.

Let us mention that in the presence of (A1), the validity of MFCQ at one point $(\bar{x}, \bar{y}) \in$ $\mathrm{gph} \Gamma$ implies that Slater's constraint qualification is valid for the set $\Gamma(\bar{x})$, i.e., there is some $\tilde{y} \in \mathbb{R}^{m}$ satisfying $h_{i}(\bar{x}, \tilde{y})<0$ for all $i \in I$ and the gradients $\left(\nabla_{y} h_{i}(\bar{x}, \cdot)\right)_{i \in J}$ (which, by validity of (A1), do not depend on $y$ ) are linearly independent. The latter, however, guarantees that MFCQ and, thus, $\operatorname{RCPLD}_{\Gamma}$ hold at each point from $\{\bar{x}\} \times \Gamma(\bar{x})$. As mentioned earlier, validity of MFCQ at $(\bar{x}, \bar{y})$ also ensures that $\bar{x}$ is an interior point of $\operatorname{dom} \Gamma$. Thus, the regularity assumptions in the first statement of Corollary 4.1 are weaker than postulating validity of MFCQ at one point from $\{\bar{x}\} \times \Gamma(\bar{x})$, and the latter is a classical assumption in the literature to guarantee local Lipschitz continuity of marginal functions, see e.g. [31, Theorem 1].

We would like to point out that the assumption on $\bar{x}$ in the first statement of Corollary 4.1 to be an interior point of $\operatorname{dom} \Gamma$ is, in general, indispensable in order to infer the local Lipschitz continuity of $\varphi$ at this point since Theorem 3.2 only provides R-regularity, and, thus, the Aubin property, of $\Gamma$ w.r.t. dom $\Gamma$. Observe that the assumptions of the second statement of Corollary 4.1 already imply that $\bar{x}$ is an interior point of $\operatorname{dom} S$.

Example 4.1 Let us consider the simple parametric optimization problem

$$
\min _{y}\{y \mid 0 \leq y \leq x\}
$$

Observing that all involved functions are fully linear, $\operatorname{RCPLD}_{\Gamma}$ holds at each point of $\mathrm{gph} \Gamma$ in this example. Nevertheless, the associated optimal value function $\varphi$ is discontinuous at 
$\bar{x}:=0$ which is a boundary point of $\operatorname{dom} \Gamma=[0, \infty)$. However, we note that $\varphi$ is Lipschitz continuous w.r.t. dom $\Gamma$.

It is also possible to obtain Lipschitzian properties of the optimal value function $\varphi$ w.r.t. $\operatorname{dom} \Gamma$ without relying on the fundamentals of variational analysis, which were used in [38], but exploiting the concept of R-regularity directly.

Lemma 4.2 Fix some point $\bar{x} \in \operatorname{dom} \Gamma$. Let one of the following additional assumptions be valid.

(a) Let (A2) hold and assume that $\Gamma$ is $R$-regular at each point from $\{\bar{x}\} \times S(\bar{x})$ w.r.t. $\operatorname{dom} \Gamma$.

(b) Assume that there exists $\bar{y} \in S(\bar{x})$ such that $\Gamma$ is $R$-regular at $(\bar{x}, \bar{y})$ w.r.t. $\operatorname{dom} \Gamma$ while $S$ is inner semicontinuous at this point w.r.t. $\operatorname{dom} \Gamma$.

Then $\varphi$ is locally Lipschitz continuous at $\bar{x}$ w.r.t. $\operatorname{dom} \Gamma$.

Proof (a) Due to $\bar{x} \in \operatorname{dom} \Gamma$ and validity of (A2), we indeed know $S(\bar{x}) \neq \varnothing$. Additionally, the set $S(\bar{x})$ is closed, i.e., $\{\bar{x}\} \times S(\bar{x})$ is compact. Thus, we can apply Lemma 2.3 in order to find constants $\kappa>0$ and $\gamma>0$ as well as an open set $O \supset S(\bar{x})$ such that (2.1) holds with $U:=\mathbb{U}_{\gamma}(\bar{x}) \times O$. Similar as in the proof of statement (c) of Lemma 4.1, we can ensure $S(x) \neq \varnothing$ for all $x \in \mathbb{U}_{\gamma}(\bar{x}) \cap \operatorname{dom} \Gamma$ if only $\gamma$ is small enough. Moreover, due to Lemma 2.2, we know that $\Gamma$ is inner semicontinuous at each point from $\{\bar{x}\} \times S(\bar{x})$ w.r.t. dom $\Gamma$. Thus, we can apply statement (b) of Lemma 4.1 in order to see that $\varphi$ is continuous at $\bar{x}$ w.r.t. $\operatorname{dom} \Gamma$. Combining this with the local boundedness of $S$ and the continuity of $h_{1}, \ldots, h_{p}$, we obtain that $S$ is upper semicontinuous at $\bar{x}$. Thus, we can even choose $\gamma$ so small that $S(x) \subset O$ holds for all $x \in \mathbb{U}_{\gamma}(\bar{x}) \cap \operatorname{dom} \Gamma$. Clearly, $\Gamma$ is upper semicontinuous at $\bar{x}$ as well which is why we find an open set $O^{\prime} \supset \Gamma(\bar{x})$ which satisfies $O^{\prime} \supset O$ and $\Gamma(x) \subset O^{\prime}$ for all $x \in \mathbb{U}_{\gamma}(\bar{x}) \cap \operatorname{dom} \Gamma$ if only $\gamma$ is sufficiently small. By continuous differentiability of the functions $f$ and $h_{1}, \ldots, h_{p}$, these functions are Lipschitz continuous on $\mathbb{B}_{\gamma}(\bar{x}) \times \operatorname{cl} O^{\prime}$. Let $L_{f}>0$ and $L_{1}, \ldots, L_{p}>0$ be the associated Lipschitz moduli.

Now, fix $x^{1}, x^{2} \in \mathbb{U}_{\gamma}(\bar{x}) \cap \operatorname{dom} \Gamma$. Then we find $y^{1}, y^{2} \in O$ such that $y^{1} \in S\left(x^{1}\right)$ and $y^{2} \in S\left(x^{2}\right)$. We exploit [11, Proposition 2.4.3] in order to see that $y^{j}$ is a global minimizer of that map $O^{\prime} \ni y \mapsto f\left(x^{j}, y\right)+2 L_{f} \operatorname{dist}\left(y, \Gamma\left(x^{j}\right)\right) \in \mathbb{R}$ for $j=1,2$ as well. Particularly, we obtain

$$
\varphi\left(x^{j}\right)=f\left(x^{j}, y^{j}\right) \leq f\left(x^{j}, y^{3-j}\right)+2 L_{f} \operatorname{dist}\left(y^{3-j}, \Gamma\left(x^{j}\right)\right), \quad j=1,2 .
$$

Now, we exploit (2.1) in order to obtain

$$
\begin{aligned}
\varphi\left(x^{1}\right) \leq & f\left(x^{1}, y^{2}\right)+2 L_{f} \operatorname{dist}\left(y^{2}, \Gamma\left(x^{1}\right)\right) \\
\leq & f\left(x^{2}, y^{2}\right)+f\left(x^{1}, y^{2}\right)-f\left(x^{2}, y^{2}\right) \\
& \quad+2 L_{f} \kappa \max \left\{0, \max \left\{h_{i}\left(x^{1}, y^{2}\right) \mid i \in I\right\}, \max \left\{\left|h_{i}\left(x^{1}, y^{2}\right)\right| \mid i \in J\right\}\right\} \\
\leq & \varphi\left(x^{2}\right)+f\left(x^{1}, y^{2}\right)-f\left(x^{2}, y^{2}\right) \\
& \quad+2 L_{f} \kappa \max \left\{0, \max \left\{h_{i}\left(x^{1}, y^{2}\right)-h_{i}\left(x^{2}, y^{2}\right) \mid i \in I\right\},\right. \\
\quad & \left.\quad \max \left\{\left|h_{i}\left(x^{1}, y^{2}\right)-h_{i}\left(x^{2}, y^{2}\right)\right| \mid i \in J\right\}\right\} \\
\leq & \varphi\left(x^{2}\right)+L_{f}\left\|x^{1}-x^{2}\right\|+2 L_{f} \kappa \max \left\{L_{i} \mid i \in I \cup J\right\}\left\|x^{1}-x^{2}\right\| \\
\leq & \varphi\left(x^{2}\right)+L_{f}\left(1+2 \kappa \max \left\{L_{i} \mid i \in I \cup J\right\}\right)\left\|x^{1}-x^{2}\right\| .
\end{aligned}
$$


Changing the roles of the pairs $\left(x^{1}, y^{1}\right)$ and $\left(x^{2}, y^{2}\right)$ yields the local Lipschitz continuity of $\varphi$ w.r.t. dom $\Gamma$.

(b) The proof can be carried out in a similar way as in (a). The postulated R-regularity of $\Gamma$ at $(\bar{x}, \bar{y})$ yields the existence of constants $\kappa>0$ as well as $\gamma>0$ and $\delta>0$ such that (3.2) holds. By inner semicontinuity of $S$ at $(\bar{x}, \bar{y})$ w.r.t. dom $\Gamma$, we can choose $\gamma$ and $\delta$ so small such that we have

$$
\forall x \in \mathbb{U}_{\gamma}(\bar{x}) \cap \operatorname{dom} \Gamma: \quad \mathbb{U}_{\delta / 2}(\bar{y}) \cap S(x) \neq \varnothing .
$$

Furthermore, we note that by continuous differentiability of $f$ and $h_{1}, \ldots, h_{p}$, these functions are Lipschitz continuous on $\mathbb{B}_{\gamma}(\bar{x}) \times \mathbb{B}_{2 \delta}(\bar{y})$ with some Lipschitz moduli $L_{f}>0$ and $L_{1}, \ldots, L_{p}>0$.

Now, fix $x^{1}, x^{2} \in \mathbb{U}_{\gamma}(\bar{x}) \cap \operatorname{dom} \Gamma$. The above arguments yield the existence of $y^{1}, y^{2} \in \mathbb{U}_{\delta / 2}(\bar{y})$ such that $y^{1} \in S\left(x^{1}\right)$ and $y^{2} \in S\left(x^{2}\right)$ hold. Exploiting [11, Proposition 2.4.3], we find

$$
\varphi\left(x^{j}\right)=f\left(x^{j}, y^{j}\right) \leq f\left(x^{j}, y^{3-j}\right)+2 L_{f} \operatorname{dist}\left(y^{3-j}, \Gamma\left(x^{j}\right) \cap \mathbb{B}_{2 \delta}(\bar{y})\right), \quad j=1,2 .
$$

Due to $y^{j} \in \Gamma\left(x^{j}\right) \cap \mathbb{U}_{\delta / 2}(\bar{y})$, we even have

$$
\operatorname{dist}\left(y^{3-j}, \Gamma\left(x^{j}\right) \cap \mathbb{B}_{2 \delta}(\bar{y})\right)=\operatorname{dist}\left(y^{3-j}, \Gamma\left(x^{j}\right)\right), \quad j=1,2,
$$

and, thus, the rest of the proof can be carried out as in statement (a).

Let us briefly mention that the first statement of the above lemma may be interpreted as an adjustment of [6, Theorem 5.4] whose set of assumptions is not complete. Indeed, in the proof of this theorem, the authors exploit the presence of R-regularity at each point from $\{\bar{x}\} \times S(\bar{x})$ which is not covered by the assumptions stated there. In [3, Theorem 4.1], the authors present criteria ensuring directional Lipschitz continuity of $\varphi$. Therefore, they impose directional R-regularity of the mapping $\Gamma$. In the non-directional case, their result essentially recovers Lemma 4.2 while exploiting a different boundedness assumption.

We obtain the following corollary from Theorems 3.2 and 3.3 as well as Lemma 4.2.

Corollary 4.2 Fix some point $\bar{x} \in \operatorname{dom} \Gamma$. Let one of the following additional assumptions be valid.

(a) Let (A1) and (A2) hold. Furthermore, let $R C P L D_{\Gamma}$ w.r.t. $\operatorname{dom} \Gamma$ hold at each point from $\{\bar{x}\} \times \Gamma(\bar{x})$.

(b) Let $\bar{y} \in S(\bar{x})$ be chosen such that $S$ is inner semicontinuous at $(\bar{x}, \bar{y})$ w.r.t. $\operatorname{dom} \Gamma$ while $R C P L D_{\Gamma}$ w.r.t. dom $\Gamma$ holds at this point.

Then $\varphi$ is locally Lipschitz continuous at $\bar{x}$ w.r.t. $\operatorname{dom} \Gamma$.

\subsubsection{R-Regularity of Solution Mappings}

The following theorem provides a sufficient criterion for R-regularity of the solution mapping $S$.

Theorem 4.1 Fix a point $\bar{x} \in \operatorname{dom} \Gamma$. Then the following assertions hold.

(a) Let (A1') and (A2) hold. Furthermore, let $R C P L D_{S}$ hold at each point from $\{\bar{x}\} \times S(\bar{x})$. Finally, let $\varphi$ be continuous at $\bar{x}$. Then $S$ is $R$-regular at each point from $\{\bar{x}\} \times S(\bar{x})$. Moreover, $S$ possesses the Aubin property at all these points. 
(b) Let $\bar{y} \in S(\bar{x})$ be chosen such that $S$ is inner semicontinuous at $(\bar{x}, \bar{y})$ while $R C P L D_{S}$ holds at this point. Then $S$ is $R$-regular at $(\bar{x}, \bar{y})$. Moreover, $S$ possesses the Aubin property at this point.

Proof We show both statements separately.

(a) Due to continuity of $\varphi$ at $\bar{x}$, we can apply Theorems 3.2 and Remark 3.1 in order to obtain R-regularity of $S$ at all points from $\{\bar{x}\} \times S(\bar{x})$. Noting that $S(\bar{x})$ is nonempty by validity of (A2), we can fix some point $y \in S(\bar{x})$. From Lemma 2.2, we infer that $S$ is inner semicontinuous at $(\bar{x}, y)$ since $\bar{x}$ is an interior point of $\operatorname{dom} S$ by continuity of $\varphi$ at $\bar{x}$. Observe that validity of $\operatorname{RCPLD}_{S}$ at $(\bar{x}, y)$ guarantees validity of $\operatorname{RCPLD}_{\Gamma}$ at this point. Now, the second statement of Corollary 4.1 ensures local Lipschitz continuity of $\varphi$ at $\bar{x}$. Consequently, locally around all points from $\{\bar{x}\} \times S(\bar{x})$, the variational description (4.1) of $S$ is given by locally Lipschitz continuous functions. Particularly, $S$ already possesses the Aubin property at all points from $\{\bar{x}\} \times S(\bar{x})$.

(b) The proof is similar to the one of the first statement. However, we exploit Theorem 3.3 to infer R-regularity of $S$ at $(\bar{x}, \bar{y})$.

The subsequently stated examples indicate that the continuity assumption in the first statement of the above theorem is, unluckily, indispensable in general since it may not follow from the postulated assumptions.

Example 4.2 Once more, let us investigate the parametric optimization problem from Example 4.1 which satisfies (A1') and (A2). There, we have

$$
\forall x \in \mathbb{R}: \quad S(x)=\left\{\begin{array}{ll}
\varnothing & x \in(-\infty, 0), \\
\{0\} & x \in[0, \infty),
\end{array} \quad \varphi(x)= \begin{cases}\infty & x \in(-\infty, 0), \\
0 & x \in[0, \infty) .\end{cases}\right.
$$

Observing that all data functions used for the modeling of the given parametric optimization problem are fully linear, $\operatorname{RCPLD}_{S}$ holds at each point from $\operatorname{gph} S$, particularly at $(\bar{x}, \bar{y}):=$ $(0,0)$. However, $\varphi$ is discontinuous at $\bar{x}$, and for $x^{k}:=-1 / k, k \in \mathbb{N}$, we obtain

$$
\operatorname{dist}\left(\bar{y}, S\left(x^{k}\right)\right)=\infty>\kappa / k=\kappa \max \left\{0, \bar{y}-\varphi\left(x^{k}\right),-\bar{y}, \bar{y}-x^{k}\right\}
$$

for each $\kappa>0$ and each $k \in \mathbb{N}$, i.e., $S$ cannot be R-regular at $(\bar{x}, \bar{y})$.

Example 4.3 We consider the parametric optimization problem

$$
\min _{y}\left\{y_{1} \mid-1 \leq y_{1} \leq 1,0 \leq y_{2} \leq 1, x y_{1}-y_{2}=0\right\} .
$$

We see that this problem inherently satisfies (A1') and (A2). The associated solution mapping $S$ and the associated marginal function $\varphi$ take the following form:

$$
\forall x \in \mathbb{R}: \quad S(x)=\left\{\begin{array}{ll}
\{(1 / x, 1)\} & x \in(-\infty,-1), \\
\{(-1,-x)\} & x \in[-1,0], \\
\{(0,0)\} & x \in(0, \infty),
\end{array} \quad \varphi(x)= \begin{cases}1 / x & x \in(-\infty,-1), \\
-1 & x \in[-1,0], \\
0 & x \in(0, \infty) .\end{cases}\right.
$$

We fix the reference points $\bar{x}:=0$ and $\bar{y}:=(-1,0)$. Clearly, $\varphi$ is not continuous at $\bar{x}$.

One can check that $\operatorname{RCPLD}_{S}$ is violated at $(\bar{x}, \bar{y})$ when using the representation of $S$ from (4.1). However, keeping Remark 4.1 in mind, we may also consider the representation

$\forall x \in \mathbb{R}: \quad S(x)=\left\{\left(y_{1}, y_{2}\right) \mid-1 \leq y_{1} \leq 1,0 \leq y_{2} \leq 1, x y_{1}-y_{2}=0, y_{1}-\varphi(x)=0\right\}$ 
of $S$ in order to address the proof of Theorem 4.1 since this representation still possesses the necessary convex structure w.r.t. $y$. One can easily check that RCPLD holds for this mapping at $(\bar{x}, \bar{y})$ since the family

$$
\left(\left(\begin{array}{l}
x \\
-1
\end{array}\right),\left(\begin{array}{l}
1 \\
0
\end{array}\right)\right)
$$

associated with the equality constraints has already constant rank 2 in a neighborhood of $(\bar{x}, \bar{y})$. However, as observed above, $\varphi$ is not continuous at $\bar{x}$, i.e., one cannot use Theorems 3.2 and Remark 3.1 in order to infer R-regularity of the solution mapping at the reference point.

Fix some point $\bar{x} \in \operatorname{dom} S$. The crucial requirement in Theorem 4.1 clearly is the validity of $\operatorname{RCPLD}_{S}$ at each or only some point from $\{\bar{x}\} \times S(\bar{x})$. As mentioned earlier, validity of MFCQ at one point from $\{\bar{x}\} \times \Gamma(\bar{x})$ is already enough to make sure that $\operatorname{RCPLD}_{\Gamma}$ holds there as well. Let us mention that, by definition of $\varphi$, there is no $y \in S(\bar{x})$ such that $h_{0}(\bar{x}, y)<0$ holds. This indicates that MFCQ generally fails to hold when applied to the variational description (4.1) of $S$ which is discussed here. Particularly, it cannot be used as a sufficient condition for $\mathrm{RCPLD}_{S}$. More details on this issue can be found in the subsequent remark.

Remark 4.2 Fix some point $(\bar{x}, \bar{y}) \in \operatorname{gph} S$. It is well known that this guarantees validity of the so-called Fritz-John conditions, i.e., we find $\lambda_{0}, \lambda_{1}, \ldots, \lambda_{p} \in \mathbb{R}$ which do not all vanish at the same time such that

$$
\begin{aligned}
& \lambda_{0} \nabla_{y} h_{0}(\bar{x}, \bar{y})+\sum_{i=1}^{p} \lambda_{i} \nabla_{y} h_{i}(\bar{x}, \bar{y})=0, \\
& \forall i \in I \cup\{0\}: \lambda_{i} \geq 0, \\
& \forall i \in I: \lambda_{i} h_{i}(\bar{x}, \bar{y})=0
\end{aligned}
$$

holds, see [7, Proposition 3.3.5]. This, however, shows that the constraint qualification MFCQ w.r.t. the representation (4.1) of the mapping $S$ cannot hold at $(\bar{x}, \bar{y})$ since the pair of families

$$
\left(\left(\nabla_{y} h_{i}(\bar{x}, \bar{y})\right)_{i \in\{0\} \cup I(\bar{x}, \bar{y})},\left(\nabla_{y} h_{i}(\bar{x}, \bar{y})\right)_{i \in J}\right)
$$

is positive-linearly dependent. Thus, versions of Theorem 4.1 which exploit MFCQ w.r.t. $S$ instead of $\operatorname{RCPLD}_{S}$ would not be reasonable at all. On the other hand, simple examples reveal that $\operatorname{RCPLD}_{S}$ can hold at $(\bar{x}, \bar{y})$, see Example 4.5 below as well.

The following lemma provides a characterization of $\operatorname{RCPLD}_{S}$ via $\operatorname{RCPLD}_{\Gamma}$.

Lemma 4.3 Fix $(\bar{x}, \bar{y}) \in \operatorname{gph} S$. Then the subsequently stated conditions are equivalent.

(a) $R C P L D_{S}$ is valid at $(\bar{x}, \bar{y})$.

(b) $R C P L D_{\Gamma}$ is valid at $(\bar{x}, \bar{y})$ with some neighborhood $U$ of $(\bar{x}, \bar{y})$ and an index set $S \subset J$ according to Definition 2.1. Furthermore, for each $\lambda \in \Lambda(\bar{x}, \bar{y})$ such that the pair of families

$$
\left(\left(\nabla_{y} h_{i}(\bar{x}, \bar{y})\right)_{i \in\{0\} \cup I_{+}(\bar{x}, \bar{y}, \lambda)},\left(\nabla_{y} h_{i}(\bar{x}, \bar{y})\right)_{i \in S}\right)
$$

is positive-linearly dependent, the family $\left(\nabla_{y} h_{i}(x, y)\right)_{i \in\{0\} \cup I_{+}(\bar{x}, \bar{y}, \lambda) \cup S}$ is linearly dependent for each $(x, y) \in U$. Above, we used

$$
\Lambda(\bar{x}, \bar{y}):=\left\{\begin{array}{l|l}
\lambda \in \mathbb{R}^{p} & \begin{array}{l}
\nabla_{y} h_{0}(\bar{x}, \bar{y})+\sum_{i=1}^{p} \lambda_{i} \nabla_{y} h_{i}(\bar{x}, \bar{y})=0, \\
\forall i \in I: \lambda_{i} \geq 0, \lambda_{i} h_{i}(\bar{x}, \bar{y})=0
\end{array}
\end{array}\right\}
$$


as well as

$$
\forall \lambda \in \Lambda(\bar{x}, \bar{y}): \quad I_{+}(\bar{x}, \bar{y}, \lambda):=\left\{i \in I(\bar{x}, \bar{y}) \mid \lambda_{i}>0\right\}
$$

Proof The implication $(\mathrm{a}) \Longrightarrow(\mathrm{b})$ is clear by definition of $\mathrm{RCPLD}_{S}$. Thus, let us assume that the conditions in (b) hold. Particularly, due to $(\bar{x}, \bar{y}) \in \operatorname{gph} S$ and validity of $\operatorname{RCPLD}_{\Gamma}$, we find $\Lambda(\bar{x}, \bar{y}) \neq \varnothing$. Fix an arbitrary index set $\tilde{K} \subset\{0\} \cup I(\bar{x}, \bar{y})$ such that the pair of families

$$
\left(\left(\nabla_{y} h_{i}(\bar{x}, \bar{y})\right)_{i \in \tilde{K}},\left(\nabla_{y} h_{i}(\bar{x}, \bar{y})\right)_{i \in S}\right)
$$

is positive-linearly dependent. In case where $\tilde{K} \subset I(\bar{x}, \bar{y})$ holds, the vectors from the family $\left(\nabla_{y} h_{i}(x, y)\right)_{i \in \tilde{K} \cup S}$ are linearly dependent for each $(x, y) \in U$ by validity of $\operatorname{RCPLD}_{\Gamma}$. Thus, we assume $0 \in \tilde{K}$. Then w.l.o.g. we find $K \subset I(\bar{x}, \bar{y})$ with $\{0\} \cup K \subset \tilde{K}$ as well as $\lambda_{i}>0(i \in\{0\} \cup K)$ and $\lambda_{i} \in \mathbb{R}(i \in S)$ satisfying

$$
\sum_{i \in\{0\} \cup K \cup S} \lambda_{i} \nabla_{y} h_{i}(\bar{x}, \bar{y})=0 .
$$

Division by $\lambda_{0}$ yields

$$
\nabla_{y} h_{0}(\bar{x}, \bar{y})+\sum_{i \in K \cup S}\left(\lambda_{i} / \lambda_{0}\right) \nabla_{y} h_{i}(\bar{x}, \bar{y})=0 .
$$

Defining $\tilde{\lambda} \in \Lambda(\bar{x}, \bar{y})$ by

$$
\forall i \in\{1, \ldots, p\}: \quad \tilde{\lambda}_{i}:= \begin{cases}\lambda_{i} / \lambda_{0} & i \in K \cup S, \\ 0 & \text { otherwise, }\end{cases}
$$

we find $K=I_{+}(\bar{x}, \bar{y}, \tilde{\lambda})$. Thus, the family $\left(\nabla_{y} h_{i}(x, y)\right)_{i \in\{0\} \cup K \cup S}$ is linearly dependent for each $(x, y) \in U$. Due to $\{0\} \cup K \subset \tilde{K}$, the family $\left(\nabla_{y} h_{i}(x, y)\right)_{i \in \tilde{K} \cup S}$ is linearly dependent as well. Consequently, $\operatorname{RCPLD}_{S}$ is valid at $(\bar{x}, \bar{y})$.

Whenever LICQ holds at $(\bar{x}, \bar{y}) \in \operatorname{gph} S$ w.r.t. the inequality and equality constraints in $\Gamma$, the criterion from Lemma 4.3 is notably easy to check since the associated Lagrange multiplier in $\Lambda(\bar{x}, \bar{y})$ is uniquely determined while $\operatorname{RCPLD}_{\Gamma}$ holds trivially. We depict this with the aid of the subsequently stated example.

Example 4.4 Let us consider the parametric optimization problem

$$
\min _{y}\left\{\left(y_{1}+1\right)^{2}+\left(y_{2}-x\right)^{2} \mid y_{1} \geq 0, y_{2} \geq 0\right\} .
$$

For later use, we set $h_{1}(x, y):=-y_{1}$ and $h_{2}(x, y):=-y_{2}$ for all $x \in \mathbb{R}$ and $y \in \mathbb{R}^{2}$. Clearly, the constraint system satisfies LICQ at each feasible point. We easily find $S(x)=$ $\{(0, \max (x, 0))\}$ for each $x \in \mathbb{R}$ as well as $\Lambda(x, y)=\{(2, \max (-2 x, 0))\}$ for each $(x, y) \in$ $\operatorname{gph} S$.

Consider $\bar{x} \geq 0$. In this case, we find $I_{+}(\bar{x}, \bar{y}, \lambda)=\{1\}$ for $\bar{y} \in S(\bar{x})$ and the associated Lagrange multiplier $\lambda \in \Lambda(\bar{x}, \bar{y})$. While the vectors in

$$
\left(\left(\begin{array}{l}
2\left(\bar{y}_{1}+1\right) \\
2\left(\bar{y}_{2}-\bar{x}\right)
\end{array}\right),\left(\begin{array}{l}
-1 \\
0
\end{array}\right)\right)
$$

are positive-linearly dependent due to $\bar{y}_{2}=\bar{x}$, a slight perturbation of $\bar{x}$ makes this family linearly independent which is why $\operatorname{RCPLD}_{S}$ fails to hold at $(\bar{x}, \bar{y})$ in this case. 
Now, fix $\bar{x}<0$. Here, we have $I_{+}(\bar{x}, \bar{y}, \lambda)=\{1,2\}$ for $\bar{y} \in S(\bar{x})$ and the associated Lagrange multiplier $\lambda \in \Lambda(\bar{x}, \bar{y})$. Noting that any strict subfamily of

$$
\left(\left(\begin{array}{l}
2\left(\bar{y}_{1}+1\right) \\
2\left(\bar{y}_{2}-\bar{x}\right)
\end{array}\right),\left(\begin{array}{l}
-1 \\
0
\end{array}\right),\left(\begin{array}{l}
0 \\
-1
\end{array}\right)\right)
$$

is linearly independent while any three vectors in $\mathbb{R}^{2}$ are linearly dependent, $\operatorname{RCPLD}_{S}$ holds at $(\bar{x}, \bar{y})$ in this case.

The subsequent remark comments on a way which allows a slight generalization of Theorem 4.1.

Remark 4.3 Let $S$ be R-regular at some point $(\bar{x}, \bar{y}) \in \operatorname{gph} S$ w.r.t. $\operatorname{dom} S$. Inspecting the proof of [6, Theorem 5.1], one only needs local Lipschitz continuity of all data functions at $(\bar{x}, \bar{y})$ w.r.t. the set $\operatorname{dom} S \times \mathbb{R}^{m}$ in order to infer validity of the Aubin property of $S$ at $(\bar{x}, \bar{y})$ w.r.t. $\operatorname{dom} S$.

Thus, the assertions of Theorem 4.1 remain true if all stated assumptions and assertions are stated w.r.t dom $\Gamma$ since this is enough to ensure local coincidence of $\operatorname{dom} S$ and $\operatorname{dom} \Gamma$. Particularly, relying on the respective second statement of Lemma 4.1 and Corollary 4.2, the requirement on $\varphi$ to be continuous at $\bar{x}$ can be removed from the assumptions which need to be postulated in the counterpart associated with the first statement of Theorem 4.1.

Keeping Lemma 2.2 and Remark 4.3 in mind, the following corollary is a direct consequence of Theorem 4.1. Indeed, this is not surprising in the light of Corollary 3.1.

Corollary 4.3 Fix a point $\bar{x} \in \operatorname{dom} \Gamma$. Let (A1') and (A2) hold. Furthermore, let RCPLDS w.r.t. $\operatorname{dom} \Gamma$ hold at each point from $\{\bar{x}\} \times S(\bar{x})$. Then $S$ is lower semicontinuous at $\bar{x}$ w.r.t. $\operatorname{dom} S$.

\subsection{Bilevel Optimization}

Let us now consider the bilevel optimization problem

$$
" \min _{x} "\{F(x, y) \mid x \in X, y \in S(x)\}
$$

where $F: \mathbb{R}^{n} \times \mathbb{R}^{m} \rightarrow \mathbb{R}$ is a continuously differentiable mapping, $X \subset \mathbb{R}^{n}$ is a closed set, and $S: \mathbb{R}^{n} \rightrightarrows \mathbb{R}^{m}$ is the solution mapping associated with $(\mathrm{P}(x))$. The model (BPP) dates back to [43] where it has been stated first in the context of economical game theory. The quotation marks in (BPP) emphasize that this problem is not necessarily well-determined. Indeed, whenever there is some $x \in X \cap \operatorname{dom} S$ where $S(x)$ is not a singleton, then the decision maker in (BPP) cannot determine the associated objective value and, thus, classical minimization is not applicable. In order to avoid this shortcoming, one often replaces (BPP) by its so-called optimistic or pessimistic version which are given by

$$
\min _{x}\left\{\varphi_{o}(x) \mid x \in X\right\} \quad \text { and } \quad \min _{x}\left\{\varphi_{p}(x) \mid x \in X\right\},
$$

respectively, where the functions $\varphi_{o}, \varphi_{p}: \mathbb{R}^{n} \rightarrow \overline{\mathbb{R}}$ are defined as follows:

$$
\forall x \in \mathbb{R}^{n}: \quad \varphi_{o}(x):=\inf _{y}\{F(x, y) \mid y \in S(x)\}, \quad \varphi_{p}(x):=\sup _{y}\{F(x, y) \mid y \in S(x)\} .
$$


This way, the optimistic and pessimistic reformulation of (BPP) reflect a cooperative behavior and a worst-case scenario between the decision makers in (BPP) and $(\mathrm{P}(x))$, respectively.

Due to numerous underlying applications, e.g., from finance, chemistry, or logistics, bilevel optimization is one of the hot topics in mathematical programming. On the other hand, (BPP) is an inherently difficult problem. Besides the above observation that it might not be well-defined, it suffers from inherent non-convexity, irregularity, and the implicit character of its feasible set. That is why numerous publications dealing with the derivation of problem-tailored optimality conditions, constraint qualifications, and solution algorithms appeared during the last three decades. We refer the interested reader to the monographs [5, $12,16]$ for a detailed introduction to bilevel optimization.

Let us take a look back at the optimistic and pessimistic version of (BPP) first. Under not too restrictive assumptions, the solution mapping $S$ is upper semicontinuous, and this property implies lower semicontinuity of $\varphi_{o}$, i.e., in case where $X$ is compact, the optimistic version of (BPP) is likely to possess a global minimizer. On the other hand, in order to guarantee lower semicontinuity of $\varphi_{p}$, one has to assume that $S$ is lower semicontinuous w.r.t. $\operatorname{dom} S$. This is quite a restrictive assumption, but our result from Corollary 4.3 depicts that it can be valid in particular problem settings. In this regard, the subsequent theorem follows from our aforementioned result and [12, Theorem 5.3].

Theorem 4.2 Let (A1') hold. Furthermore, assume that $X \subset \operatorname{dom} \Gamma$ holds true and that $\Gamma$ is locally bounded at each point from X. Additionally, let $R C P L D_{S}$ w.r.t. $\operatorname{dom} \Gamma$ hold at each point from gphS $\cap\left(X \times \mathbb{R}^{m}\right)$. Finally, let $X$ be nonempty and compact. Then there exists $a$ pessimistic solution of (BPP).

The crucial requirement in the above theorem obviously is the validity of $\operatorname{RCPLD}_{S}$ w.r.t. $\operatorname{dom} \Gamma$ at each point from $\operatorname{gph} S \cap\left(X \times \mathbb{R}^{m}\right)$, see Lemma 4.3 and the subsequent comments for some discussion. However, let us note that this is inherent for lower level problems of type

$$
\min _{y}\left\{c^{\top} y \mid B y \leq b(x)\right\}
$$

where $c \in \mathbb{R}^{m}$ and $B \in \mathbb{R}^{\ell \times m}$ are matrices while $b: \mathbb{R}^{n} \rightarrow \mathbb{R}^{\ell}$ is a continuous function. This means that (BPP) with the special lower level problem (4.2) is likely to possess a pessimistic solution.

Observing that the optimistic and pessimistic version of (BPP) might be interpreted as a three-level decision process, the derivation of optimality conditions via these models is quite challenging, see e.g. [17, 18]. In the literature, it is a common approach to consider

$$
\min _{x, y}\{F(x, y) \mid x \in X, y \in S(x)\}
$$

instead. This well-defined optimization problem is closely related to the optimistic version of (BPP), see [17, Proposition 6.9] for details. Furthermore, by definition of the optimal value function, one can easily check that $\left(\mathrm{BPP}^{\prime}\right)$ is fully equivalent to the single-level optimization problem

$$
\min _{x, y}\{F(x, y) \mid x \in X, f(x, y)-\varphi(x) \leq 0, y \in \Gamma(x)\}
$$

which is commonly referred to as the optimal value reformulation or value function transformation of $\left(\mathrm{BPP}^{\prime}\right)$. Although this problem is still quite challenging due to the implicit character of $\varphi$, the general non-smoothness of $\varphi$, and its inherent irregularity, it has been 
exploited intensively for the derivation of necessary optimality conditions and solution algorithms, see e.g. [13-15, 19, 23, 39, 46, 47] and the references therein. The key idea in all these papers is to use a partial penalization argument in order to shift the crucial constraint $f(x, y)-\varphi(x) \leq 0$ from the feasible set of (OVR) to its objective function. Whenever this penalization is locally exact, this approach is reasonable in theory and numerical practice. Following [46], we refer to this property as partial calmness.

Definition 4.1 Let $(\bar{x}, \bar{y}) \in \mathbb{R}^{n} \times \mathbb{R}^{m}$ be a locally optimal solution of $\left(\mathrm{BPP}^{\prime}\right)$. We say that this program is partially calm at $(\bar{x}, \bar{y})$ if there exist a neighborhood $U$ of $(\bar{x}, \bar{y}, 0)$ and some constant $\kappa>0$ such that we have $F(x, y)-F(\bar{x}, \bar{y})+\kappa|u| \geq 0$ for each triplet $(x, y, u) \in U$ which satisfies

$$
x \in X, \quad f(x, y)-\varphi(x) \leq u, \quad y \in \Gamma(x) .
$$

Indeed, [46, Proposition 3.3] shows that ( $\left.\mathrm{BPP}^{\prime}\right)$ is partially calm at one of its local minimizers $(\bar{x}, \bar{y})$ if and only if there is some $\kappa>0$ such that $(\bar{x}, \bar{y})$ is a local minimizer of

$$
\min _{x, y}\{F(x, y)+\tilde{\kappa}(f(x, y)-\varphi(x)) \mid x \in X, y \in \Gamma(x)\}
$$

for each $\tilde{\kappa} \geq \kappa$. Noting that the latter optimization problem may satisfy standard constraint qualifications, the presence of partial calmness indeed opens a way to the derivation of necessary optimality conditions for $\left(\mathrm{BPP}^{\prime}\right)$ since the potential non-smoothness of $\varphi$ now can be simply handled with suitable subdifferential constructions from variational analysis.

In [33, Section 3], the authors provide an overview of conditions which are sufficient for the presence of partial calmness in bilevel optimization. Our particular interest here lies in a result which can be distilled from [33, Lemmas 3.2 and 3.3].

Proposition 4.1 Let $(\bar{x}, \bar{y}) \in \mathbb{R}^{n} \times \mathbb{R}^{m}$ be a local minimizer of (BPP') such that $S$ is $R$ regular at $(\bar{x}, \bar{y})$ w.r.t. dom $S$. Furthermore, assume that the sets dom $\Gamma$ and $\operatorname{dom} S$ coincide locally around $\bar{x}$. Then $\left(\mathrm{BPP}^{\prime}\right)$ is partially calm at $(\bar{x}, \bar{y})$.

We would like to point out that a related result can be found in [6, Theorem 6.1].

As mentioned in [33, Lemma 3.3], the assumptions of Proposition 4.1 actually imply that the point $(\bar{x}, \bar{y})$ corresponds to a so-called (local) unifomly weak sharp minimum of the parametric optimization problem $(\mathrm{P}(x))$, and the latter guarantees partial calmness of (BPP') at $(\bar{x}, \bar{y})$, see [46, Proposition 5.1] as well. However, while the presence of a uniformly weak sharp minimum is generally hard to verify by definition, the assumptions of Proposition 4.1 can be established, e.g., using the results of Section 4.1 and, thus, in terms of initial data.

Consequently, we may apply Theorem 4.1 as well as Remark 4.3 in order to infer new sufficient conditions for the validity of partial calmness.

Theorem 4.3 Let $(\bar{x}, \bar{y}) \in \mathbb{R}^{n} \times \mathbb{R}^{m}$ be a local minimizer of $\left(\mathrm{BPP}^{\prime}\right)$. Additionally, let one of the following additional conditions hold.

(a) Let (A1') and (A2) be valid. Furthermore, let RCPLD $D_{S}$ w.r.t. dom $\Gamma$ hold at each point from $\{\bar{x}\} \times S(\bar{x})$.

(b) Let $S$ be inner semicontinuous at $(\bar{x}, \bar{y})$ w.r.t. $\operatorname{dom} \Gamma$ and let $R C P L D_{S}$ w.r.t. $\operatorname{dom} \Gamma$ hold at this point.

Then $\left(\mathrm{BPP}^{\prime}\right)$ is partially calm at $(\bar{x}, \bar{y})$. 
As we already observed above, the crucial assumption $\operatorname{RCPLD}_{S}$ is generally valid for lower level problems of type (4.2) which is why the local minimizers of the associated bilevel optimization problem $\left(\mathrm{BPP}^{\prime}\right)$ are always partially calm. This observation already has been made in [33, Theorem 4.1] and [34, Lemma 2.1]. However, we would like to point out that our result from Theorem 4.3 may address far more general situations as demonstrated with the aid of the subsequent example.

Example 4.5 Let us consider the bilevel optimization problem

$$
\min _{x, y}\left\{(x-3 / 4)^{2}+y^{2} \mid y \in S(x)\right\}
$$

where $S: \mathbb{R} \rightrightarrows \mathbb{R}$ is the solution mapping of the parametric optimization problem

$$
\min _{y}\left\{(x+y-2)^{2} \mid y^{2}-x \leq 0, y \geq 0\right\} .
$$

Some computations show

$$
\forall x \in \mathbb{R}: \quad S(x)=\left\{\begin{array}{ll}
\varnothing & x \in(-\infty, 0), \\
\{\sqrt{x}\} & x \in[0,1], \\
\{2-x\} & x \in[1,2], \\
\{0\} & x \in[2, \infty),
\end{array} \quad \varphi(x)= \begin{cases}\infty & x \in(-\infty, 0), \\
(x+\sqrt{x}-2)^{2} & x \in[0,1], \\
0 & x \in[1,2], \\
(x-2)^{2} & x \in[2, \infty) .\end{cases}\right.
$$

We observe that $S$ is a single-valued and continuous map w.r.t. its domain. Particularly, it is inner semicontinuous w.r.t. $\operatorname{dom} S$ at each point of its graph. Furthermore, $\operatorname{dom} S=\operatorname{dom} \Gamma$ holds. Using the above formula for $S$, one can easily check that (4.3) possesses the uniquely determined global minimizer $(\bar{x}, \bar{y}):=(1 / 4,1 / 2)$ while there is another local minimizer at $(\tilde{x}, \tilde{y}):=(11 / 8,5 / 8)$.

We observe that each subsystem of the family $(2(x+y-2), 2 y)$ possesses constant rank around the reference point $(\bar{x}, \bar{y})$, and this is sufficient for the validity of $\operatorname{RCPLD}_{S}$ at $(\bar{x}, \bar{y})$, i.e., (4.3) is partially calm at this point by Theorem 4.3.

Next, we consider the point $(\tilde{x}, \tilde{y})$. Here, the set of lower level active constraints is empty and the gradient of the lower level objective function vanishes but, clearly, does not generally vanish in a neighborhood of $(\tilde{x}, \tilde{y})$. Thus, $\operatorname{RCPLD}_{S}$ is violated at $(\tilde{x}, \tilde{y})$, i.e., we cannot employ Theorem 4.3 in order to infer partial calmness of $(4.3)$ at $(\tilde{x}, \tilde{y})$. However, one can easily check that, for each $\kappa>0,(\tilde{x}, \tilde{y})$ is not a local minimizer of

$$
\min _{x, y}\left\{(x-3 / 4)^{2}+y^{2}+\kappa\left((x+y-2)^{2}-\varphi(x)\right) \mid y^{2}-x \leq 0, y \geq 0\right\}
$$

(note that, locally around $(\tilde{x}, \tilde{y})$, this is a convex problem) which is why (4.3) is actually not partially calm at $(\tilde{x}, \tilde{y})$.

\section{Conclusions}

In this manuscript, we have shown that the validity of the constraint qualification RCPLD is sufficient to infer the presence of R-regularity for set-valued mappings of type (1.1). Our results generalize similar considerations which exploit the constraint qualifications MFCQ or RCRCQ for that purpose, see [6,32,36]. We applied our findings in order to study nonlinear parametric optimization problems and bilevel optimization problems. First, we inferred new criteria ensuring Lipschitz continuity of optimal value functions as well as Rregularity and lower semicontinuity of solution mappings in parametric programming. As we have seen, a similar analysis w.r.t. the solution mapping is not possible under MFCQ. 
Second, these results were exploited in order to state a criterion for the existence of solutions in pessimistic bilevel optimization as well as a sufficient condition for the validity of the partial calmness property in optimistic bilevel optimization. Throughout the manuscript, simple examples illustrated applicability but also the limits of our findings.

Funding Open Access funding enabled and organized by Projekt DEAL.

Open Access This article is licensed under a Creative Commons Attribution 4.0 International License, which permits use, sharing, adaptation, distribution and reproduction in any medium or format, as long as you give appropriate credit to the original author(s) and the source, provide a link to the Creative Commons licence, and indicate if changes were made. The images or other third party material in this article are included in the article's Creative Commons licence, unless indicated otherwise in a credit line to the material. If material is not included in the article's Creative Commons licence and your intended use is not permitted by statutory regulation or exceeds the permitted use, you will need to obtain permission directly from the copyright holder. To view a copy of this licence, visit http://creativecommons.org/licenses/by/4.0/.

\section{References}

1. Andreani, R., Haeser, G., Schuverdt, M.L., Silva, P.J.S.: A relaxed constant positive linear dependence constraint qualification and applications. Math. Program. 135(1), 255-273 (2012). https://doi.org/10.1007/s10107-011-0456-0

2. Andreani, R., Martinez, J.M., Schuverdt, M.L.: On the relation between constant positive linear dependence condition and quasinormality constraint qualification. J. Optim. Theory Appl. 125(2), 473-483 (2005). https://doi.org/10.1007/s10957-004-1861-9

3. Bai, K., Ye, J.J.: Directional necessary optimality conditions for bilevel programs. arXiv:2004.01783,131 (2020)

4. Bank, B., Guddat, J., Klatte, D., Kummer, B., Tammer, K.: Nonlinear Parametric Optimization. Basel, Birkhäuser (1983)

5. Bard, J.F.: Practical Bilevel Optimization: Algorithms and Applications. Kluwer Academic, Dordrecht (1998)

6. Bednarczuk, E.M., Minchenko, L.I., Rutkowski, K.E.: On Lipschitz-like continuity of a class of set-valued mappings. Optimization 69(12), 2535-2549 (2020). https://doi.org/10.1080/02331934.2019.1696339

7. Bertsekas, D.P.: Nonlinear Programming. Athena Scientific, Belmot (1999)

8. Borwein, J.M.: Stability and regular points of inequality systems. J. Optim. Theory Appl. 48(1), 9-52 (1986). https://doi.org/10.5555/3182697.3183275

9. Bosch, P., Jourani, A., Henrion, R.: Sufficient conditions for error bounds and applications. Appl. Math. Optim. 50(2), 161-181 (2004). https://doi.org/10.1007/s00245-004-0799-5

10. Chieu, N.H., Lee, G.M.: A relaxed constant positive linear dependence constraint qualification for mathematical programs with equilibrium constraints. J. Optim. Theory Appl. 158(1), 11-32 (2013). https://doi.org/10.1007/s10957-012-0227-y

11. Clarke, F.H.: Optimization and Nonsmooth Analysis. Wiley, New York (1983)

12. Dempe, S.: Foundations of Bilevel Programming. Kluwer, Dordrecht (2002)

13. Dempe, S., Dutta, J., Mordukhovich, B.S.: New necessary optimality conditions in optimistic bilevel programming. Optimization 56(5-6), 577-604 (2007). https://doi.org/10.1080/02331930701617551

14. Dempe, S., Franke, S.: The bilevel road pricing problem. Int. J. Comput. Optim. 2(2), 71-92 (2015). https://doi.org/10.12988/ijco.2015.5415

15. Dempe, S., Franke, S.: On the solution of convex bilevel optimization problems. Comput. Optim. Appl. 63(3), 685-703 (2016). https://doi.org/10.1007/s10589-015-9795-8

16. Dempe, S., Kalashnikov, V., Pérez-Valdéz, G., Kalashnykova, N.: Bilevel Programming Problems Theory, Algorithms and Applications to Energy Networks. Springer, Berlin (2015)

17. Dempe, S., Mordukhovich, B.S., Zemkoho, A.B.: Sensitivity analysis for two-level value functions with applications to bilevel programming. SIAM J. Optim. 22(4), 1309-1343 (2012). https://doi.org/10.1137/110845197

18. Dempe, S., Mordukhovich, B.S., Zemkoho, A.B.: Necessary optimality conditions in pessimistic bilevel programming. Optimization 63(4), 505-533 (2014). https://doi.org/10.1080/02331934.2012.696641 
19. Dempe, S., Zemkoho, A.B.: The bilevel programming problem: reformulations, constraint qualifications and optimality conditions. Math. Program. 138(1), 447-473 (2013). https://doi.org/10.1007/s10107-011-0508-5

20. Fabian, M.J., Henrion, R., Kruger, A.Y., Outrata, J.V.: Error bounds: necessary and sufficient conditions. Set-Valued Variat. Anal. 18(2), 121-149 (2010). https://doi.org/10.1007/s11228-010-0133-0

21. Fedorov, V.V.: Numerical Maximin Methods. Moscow, Nauka (1979)

22. Fiacco, A.V.: Optimal Value Continuity and Differential Stability Bounds under the MangasarianFromovitz Constraint Qualification. In: Fiacco, A.V. (ed.) Mathematical Programming with Data Perturbations, vol. 2, pp. 65-90. Marcel Dekker, New York (1983)

23. Fischer, A., Zemkoho, A.B., Zhou, S.: Semismooth Newton-type method for bilevel optimization: global convergence and extensive numerical experiments. arXiv:1912.07079, 1-27 (2019)

24. Gfrerer, H., Mordukhovich, B.S.: Robinson stability of parametric constraint systems via variational analysis. SIAM J. Optim. 27(1), 438-465 (2017). https://doi.org/10.1137/16M1086881

25. Gfrerer, H., Outrata, J.V.: On Lipschitzian properties of implicit multifunctions. SIAM J. Optim. 26(4), 2160-2189 (2016). https://doi.org/10.1137/15M1052299

26. Guo, L., Lin, G.H.: Notes on some constraint qualifications for mathematical programs with equilibrium constraints. J. Optim. Theory Appl. 156, 600-616 (2013). https://doi.org/10.1007/s10957-012-0084-8

27. Guo, L., Lin, G.H., Ye, J.J.: Second-order optimality conditions for mathematical programs with equilibrium constraints. J. Optim. Theory Appl. 158, 33-64 (2013). https://doi.org/10.1007/s10957-012-0228-x

28. Ioffe, A.D.: Regular points of Lipschitz functions. Trans. Am. Math. Soc. 251, 61-69 (1979). https://doi.org/10.1090/S0002-9947-1979-0531969-6

29. Ioffe, A.D.: Metric regularity and subdifferential calculus. Russ. Math. Surv. 55(3), 501-558 (2000). https://doi.org/10.1070/RM2000v055n03ABEH000292

30. Janin, R.: Directional Derivative of the Marginal Function in Nonlinear Programming. In: Fiacco, A.V. (ed.) Sensitivity, Stability and Parametric Analysis, vol. 21, pp. 110-126. Springer, Berlin (1984). https://doi.org/10.1007/BFb0121214

31. Klatte, D., Kummer, B.: Stability Properties of Infima and Optimal Solutions of Parametric Optimization Problems. In: Demyanov, V.F., Pallaschke, D. (eds.) Nondifferentiable Optimization: Motivations and Applications, pp. 215-229. Springer, Berlin (1985)

32. Luderer, B., Minchenko, L.I., Satsura, T.: Multivalued Analysis and Nonlinear Programming Problems with Perturbations. Springer Science+Business Media, Dordrecht (2002)

33. Mehlitz, P., Minchenko, L.I., Zemkoho, A.B.: A note on partial calmness for bilevel optimization problems with linearly structured lower level. Optim. Lett., 1-15. https://doi.org/10.1007/s11590-020-01636-6 (2020)

34. Minchenko, L.I., Berezhnov, D.E.: On global partial calmness for bilevel programming problems with linear lower-level problem. In: CEUR Workshop Proceedings, vol. 1987 (2017). http://ceur-ws.org/ Vol-1987/paper60.pdf

35. Minchenko, L.I., Stakhovski, S.: On relaxed constant rank regularity condition in mathematical programming. Optimization 60(4), 429-440 (2011). https://doi.org/10.1080/02331930902971377

36. Minchenko, L.I., Stakhovski, S.: Parametric nonlinear programming problems under the relaxed constant rank condition. SIAM J. Optim. 21(1), 314-332 (2011). https://doi.org/10.1137/090761318

37. Mordukhovich, B.S.: Variational Analysis and Generalized Differentiation I: Basic Theory. II Applications. Springer, Berlin (2006)

38. Mordukhovich, B.S., Nam, N.M.: Variational stability and marginal functions via generalized differentiation. Math. Oper. Res. 30(4), 800-816 (2005). https://doi.org/10.1287/moor.1050.0147

39. Mordukhovich, B.S., Nam, N.M., Phan, H.M.: Variational analysis of marginal functions with applications to bilevel programming. J. Optim. Theory Appl. 152(3), 557-586 (2012). https://doi.org/10.1007/s10957-011-9940-1

40. Qi, L., Wei, Z.: On the constant positive linear dependence condition and its application to SQP methods. SIAM J. Optim. 10(4), 963-981 (2000). https://doi.org/10.1137/S1052623497326629

41. Robinson, S.M.: Stability theory for systems of inequalities, part II: differentiable nonlinear systems. SIAM J. Numer. Anal. 13(4), 497-513 (1976)

42. Rockafellar, R.T., Wets, R.J.B.: Variational Analysis Grundlehren Der Mathematischen Wissenschaften, vol. 317. Springer, Berlin (1998)

43. v.Stackelberg, H.: Marktform und Gleichgewicht. Springer, Berlin (1934)

44. $\mathrm{Xu}, \mathrm{M}$., Ye, J.J.: Relaxed constant positive linear dependence constraint qualification and its application to bilevel programs. J. Glob. Optim. 78(1), 181-205 (2020). https://doi.org/10.1007/s10898-020-00907-X

45. Ye, J.J.: New uniform parametric error bounds. J. Optim. Theory Appl. 98, 197-219 (1998). https://doi.org/10.1023/A:1022649217032 
46. Ye, J.J., Zhu, D.L.: Optimality conditions for bilevel programming problems. Optimization 33(1), 9-27 (1995). https://doi.org/10.1080/02331939508844060

47. Ye, J.J., Zhu, D.L.: New necessary optimality conditions for bilevel programs by combining the MPEC and value function approaches. SIAM J. Optim. 20(4), 1885-1905 (2010). https://doi.org/10.1137/080725088

48. Yen, N.D.: Stability of the solution set of perturbed nonsmooth inequality systems and applications. J. Optim. Theory Appl. 93, 199-225 (1997). https://doi.org/10.1023/A:1022662120550

Publisher's Note Springer Nature remains neutral with regard to jurisdictional claims in published maps and institutional affiliations. 